TRANSACTIONS OF THE

AMERICAN MATHEMATICAL SOCIETY

Volume 352, Number 9, Pages 4113-4141

S 0002-9947(99)02489-7

Article electronically published on October 5, 1999

\title{
ON THE HOMOTOPY OF SIMPLICIAL ALGEBRAS OVER AN OPERAD
}

\author{
BENOIT FRESSE
}

\begin{abstract}
According to a result of H. Cartan, the homotopy of a simplicial commutative algebra is equipped with divided power operations. In this article, we show how to extend this result to other kinds of algebras. For instance, we prove that the homotopy of a simplicial Lie algebra is equipped with the structure of a restricted Lie algebra.
\end{abstract}

\section{INTRODUCTION}

According to a result of H. Cartan (cf. [5]), the homotopy of a simplicial commutative algebra is equipped with divided power operations. In this paper, we provide a general approach to the construction of such operations in the context of simplicial algebras over an operad. To be precise, we work over a fixed field $\mathbf{F}$, and we consider operads in the category of $\mathbf{F}$-modules. An operad is an algebraic device that specifies a type of algebras. There are operads $\mathcal{C}$ om, $\mathcal{A} s, \mathcal{L} i e$, and $\mathcal{P}$ ois, whose algebras are respectively commutative algebras, associative algebras, Lie algebras and Poisson algebras. In general, if $\mathcal{P}$ denotes an operad, then we call $\mathcal{P}$-algebras the associated algebras.

First, we generalize the notion of a divided power in the context of algebras over an operad. This is done as follows. Recall that the free commutative algebra is given by the formula

$$
T(\mathcal{C o m}, V)=\bigoplus_{n}\left(V^{\otimes n}\right)_{S_{n}} \quad \text { for } V \in \operatorname{Mod}_{\mathbf{F}} .
$$

If we replace the coinvariants by the invariants in this construction, then it is known that we obtain the free divided power algebra

$$
\Gamma(\mathcal{C o m}, V)=\bigoplus_{n}\left(V^{\otimes n}\right)^{S_{n}} \quad \text { for } V \in \operatorname{Mod}_{\mathbf{F}} .
$$

Now, an operad $\mathcal{P}$ consists of a sequence of $S_{n}$-modules $\mathcal{P}(n)$ equipped with a certain product structure. The free $\mathcal{P}$-algebra $T(\mathcal{P}, V)$ has the following expansion:

$$
T(\mathcal{P}, V)=\bigoplus_{n}\left(\mathcal{P}(n) \otimes V^{\otimes n}\right)_{S_{n}} \quad \text { for } V \in \operatorname{Mod}_{\mathbf{F}} .
$$

Equivalently, the functor $T(\mathcal{P},-)$ defined by the formula above is a monad which has the $\mathcal{P}$-algebras as associated algebras. We show that the functor $\Gamma(\mathcal{P},-)$ defined

Received by the editors February 17, 1998.

1991 Mathematics Subject Classification. Primary 18C15, 18G30; Secondary 55S99, 55U10.

(C)2000 American Mathematical Society 
by

$$
\Gamma(\mathcal{P}, V)=\bigoplus_{n}\left(\mathcal{P}(n) \otimes V^{\otimes n}\right)^{S_{n}} \quad \text { for } V \in \operatorname{Mod}_{\mathbf{F}},
$$

is also equipped with a monad structure. We call $\mathcal{P}$-algebra with divided symmetries (or $\Gamma \mathcal{P}$-algebra for short) an algebra over the $\operatorname{monad} \Gamma(\mathcal{P},-)$. This notion generalizes the structure of a divided power algebra. In fact, by the observation recalled above, a $\Gamma \mathcal{C}$ om-algebra is nothing but a divided power algebra. We determine explicitly the structure of a $\Gamma \mathcal{P}$-algebra for the other classical operads $\mathcal{P}=\mathcal{A} s, \mathcal{L}$ ie, Pois. We prove the following main result (see theorem 1.2.5 for a more precise statement):

0.1. Theorem. Fix a ground field $\mathbf{F}$ of characteristic $p$. Let $\mathcal{L}$ ie denote the Lie operad. Hence, the free Lie algebra has the expansion

$$
T(\mathcal{L} i e, V)=\bigoplus_{n}\left(\mathcal{L} i e(n) \otimes V^{\otimes n}\right)_{S_{n}} .
$$

We claim that the functor

$$
\Gamma(\mathcal{L} i e, V)=\bigoplus_{n}\left(\mathcal{L} i e(n) \otimes V^{\otimes n}\right)^{S_{n}}
$$

represents the free p-restricted Lie algebra.

This theorem does not seem to occur in the literature. It implies that a $\Gamma \mathcal{L} i e$ algebra is a $p$-restricted Lie algebra. In this sense, $p$-restricted Lie algebras play the same role with respect to Lie algebras as divided power algebras with respect to commutative algebras. In the case of the associative operad, we have

$$
T(\mathcal{A} s, V)=\Gamma(\mathcal{A} s, V)=\bigoplus_{n} V^{\otimes n}
$$

$\left(\mathcal{A} s(n)\right.$ is the regular representation $\left.\mathbf{F}\left[S_{n}\right]\right)$. It follows that a $\Gamma \mathcal{A} s$-algebra does not carry more structure than an associative algebra.

Now, we can state our generalization of Cartan's result.

0.2. Theorem. Let $A$ be a 2-reduced simplicial $\mathcal{P}$-algebra (i.e., $A_{0}=A_{1}=0$ ). The homotopy of $A$, which is the homology of the associated complex, is equipped with a structure of a graded $\Gamma \mathcal{P}$-algebra.

The results of this article were announced in 13.

Acknowledgements. I am grateful to J. McCleary for his careful reading of the preliminary manuscript and to V. Franjou for enlightened remarks. I thank the referee for his careful reading of the present article which helped us to eliminate some significant inaccuracies from the final version.

Summary. 1. Algebras with divided symmetries

1.1. Operads and associated monads. In this section, we recall the basics of operad theory, and in parallel, we define the notion of a $\Gamma \mathcal{P}$-algebra.

1.2. Examples. We write down the structure of a $\Gamma \mathcal{P}$-algebra, when $\mathcal{P}$ is one of the classical operads cited above, $\mathcal{A} s, \mathcal{C}$ om, $\mathcal{L} i e, \mathcal{P}$ ois.

2. Homotopy operations

2.1. General theory and classical results. We recall some classical results on the nonlinear derived functors of Dold and Puppe. 
2.2. Homotopy operations for simplicial algebras over an operad. In this paper, we are concerned with $T(\mathcal{P})_{*}$, the derived functor of the monad $T(\mathcal{P},-)$ associated to an operad $\mathcal{P}$. The functor $T(\mathcal{P})_{*}$ is equipped with a canonical monad structure and the homotopy of a simplicial $\mathcal{P}$-algebra is an algebra over this monad $T(\mathcal{P})_{*}$. We construct an embedding $\Gamma(\mathcal{P}, V) \hookrightarrow T(\mathcal{P})_{*}(V)$ and theorem 0.2 follows.

\section{Notation and conventions.}

0.3. Ground categories. Throughout this paper we work over a fixed field $\mathbf{F}$ of characteristic $p$. We denote by $\operatorname{Mod}_{\mathbf{F}}$ the category of $\mathbf{F}$-modules, by $s \operatorname{Mod}_{\mathbf{F}}$ the category of simplicial $\mathbf{F}$-modules and by $n \operatorname{Mod}_{\mathbf{F}}$ the category of positively graded F-modules. We denote by $\mathcal{F}\left(\operatorname{Mod}_{\mathbf{F}}\right)$ the category of endofunctors of $\operatorname{Mod}_{\mathbf{F}}$. If $x$ is a homogeneous element in a graded module, then $|x|$ denotes the degree of $x$.

0.4. Permutations. We denote the symmetric group by $S_{n}$. Let $\alpha=\left(\alpha_{1}, \ldots, \alpha_{r}\right)$ be an $r$-tuple of non-negative integers such that $n=\alpha_{1}+\cdots+\alpha_{r}$. We denote by $S_{\alpha}$ the Young subgroup of $S_{n}$

$$
S_{\alpha_{1}} \times \cdots \times S_{\alpha_{r}} \longleftrightarrow S_{n}
$$

A permutation of $S_{n}$ which is the direct product of $\sigma_{1} \in S_{\alpha_{1}}, \ldots, \sigma_{r} \in S_{\alpha_{r}}$, is denoted by $\sigma_{1} \oplus \cdots \oplus \sigma_{r}$. If $s \in S_{r}$, then $s\left(\alpha_{1}, \ldots, \alpha_{r}\right)$ denotes the block permutation of $S_{n}$, which is defined by

$$
\begin{aligned}
s\left(\alpha_{1}, \ldots, \alpha_{r}\right)\left(\alpha_{s(1)}+\cdots+\alpha_{s(l-1)}+k\right):=\alpha_{1}+\cdots+\alpha_{s(l)-1}+k, & \\
k & =1, \ldots, \alpha_{s(l)}, l=1, \ldots, r .
\end{aligned}
$$

In particular, if $\tau$ denotes the transposition of $S_{2}$, then $\tau(i, j) \in S_{n}$ is the block transposition:

$$
\begin{array}{ll}
\tau(i, j)(k)=i+k, & k=1, \ldots, j, \\
\tau(i, j)(j+k)=k, & k=1, \ldots, i .
\end{array}
$$

Let $M_{i}, i=1, \ldots, r$, be an $S_{\alpha_{i}}$-module. The tensor product $M_{1} \otimes \cdots \otimes M_{r}$ is an $S_{\alpha}$-module. We may identify the tensor $x_{1} \otimes \cdots \otimes x_{r} \in M_{1} \otimes \cdots \otimes M_{r}$ with its image in the induced module $\operatorname{Ind}_{S_{\alpha}}^{S_{n}} M_{1} \otimes \cdots \otimes M_{r}$. Moreover, if $\sigma \in S_{n}$, then $\sigma \cdot x_{1} \otimes \cdots \otimes x_{r}$ denotes the action of $\sigma$ on $x_{1} \otimes \cdots \otimes x_{r}$ in $\operatorname{Ind}_{S_{\alpha}}^{S_{n}} M_{1} \otimes \cdots \otimes M_{r}$. Hence, $\sigma \cdot x_{1} \otimes \cdots \otimes x_{r}$ is the tensor

$$
\sigma \otimes x_{1} \otimes \cdots \otimes x_{r} \in \mathbf{F}\left[S_{n}\right] \otimes \mathbf{F}\left[S_{\alpha}\right] M_{1} \otimes \cdots \otimes M_{r}=\operatorname{Ind}_{S_{\alpha}}^{S_{n}} M_{1} \otimes \cdots \otimes M_{r} .
$$

0.5. Place permutation. Let $V$ be an F-module. The symmetric group $S_{r}$ acts on $V^{\otimes r}$ by place permutation. If $v_{1} \otimes \cdots \otimes v_{r} \in V^{\otimes r}$, then we denote

$$
\begin{aligned}
& s^{*}\left(v_{1} \otimes \cdots \otimes v_{r}\right):=v_{s(1)} \otimes \cdots \otimes v_{s(r)}, \\
& s_{*}\left(v_{1} \otimes \cdots \otimes v_{r}\right):=v_{s^{-1}(1)} \otimes \cdots \otimes v_{s^{-1}(r)} .
\end{aligned}
$$

If $V$ is graded, then we insert the appropriate signs in these formulas.

0.6. Operads. In the sequel, an operad denotes an operad in the monoidal category of $\mathbf{F}$-modules $\left(\operatorname{Mod}_{\mathbf{F}}, \otimes\right)$ (cf. 1.1.10). Furthermore, any operad $\mathcal{P}$ is tacitly assumed to be connected (explicitly, $\mathcal{P}(0)=0$ ). We use the language of monads (following the terminology of [23] Chapter VI]). In the literature, a monad is also known as a triple (cf. [1, Chapter 3]). 


\section{Algebras With Divided Symmetries}

\subsection{Operads and associated monads.}

1.1.1. On symmetric sequences and associated functors. A symmetric sequence of $\mathbf{F}$-modules $M$ is a sequence of $S_{n}$-modules $M(n), n \geq 0$. We say that $M$ is connected if $M(0)=0$. A morphism of symmetric sequences $f: M \longrightarrow N$ is a sequence of morphisms of $S_{n}$-modules $f: M(n) \longrightarrow N(n)$. We denote the category of symmetric sequences by $\operatorname{Mod}_{\mathbf{F}}^{S}$. The notation $x \in M(n)$ may be abbreviated to $x \in M$. The degree of $x$, denoted by $|x|$, is the integer $n$. In this article, we follow the terminology of [20]. A symmetric sequence is also known as a collection (cf. [16]), and as an S-module (cf. [15, 14]).

Recall the definition of the functors $T(M,-): \operatorname{Mod}_{\mathbf{F}} \longrightarrow \operatorname{Mod}_{\mathbf{F}}$ and $\Gamma(M,-)$ : $\operatorname{Mod}_{\mathbf{F}} \longrightarrow \operatorname{Mod}_{\mathbf{F}}$. Let $V$ be an $\mathbf{F}$-module. We set

$$
T(M, V):=\bigoplus_{n \geq 0}\left(M(n) \otimes V^{\otimes n}\right)_{S_{n}}
$$

and

$$
\Gamma(M, V):=\bigoplus_{n \geq 0}\left(M(n) \otimes V^{\otimes n}\right)^{S_{n}} .
$$

Observe that one can extend $T(M,-)$ and $\Gamma(M,-)$ to the category of graded $\mathbf{F}$ modules $n \operatorname{Mod}_{\mathbf{F}}$.

Recall that $\mathcal{F}\left(\operatorname{Mod}_{\mathbf{F}}\right)$ denotes the category of endofunctors of $\operatorname{Mod}_{\mathbf{F}}$. Clearly, we have functors

$$
T: \operatorname{Mod}_{\mathbf{F}}^{S} \longrightarrow \mathcal{F}\left(\operatorname{Mod}_{\mathbf{F}}\right) \quad \text { and } \quad \Gamma: \operatorname{Mod}_{\mathbf{F}}^{S} \longrightarrow \mathcal{F}\left(\operatorname{Mod}_{\mathbf{F}}\right),
$$

such that $T(M)(V):=T(M, V)$ and $\Gamma(M)(V):=\Gamma(M, V)$. These functors $T$ and $\Gamma$ are faithful. But, in positive characteristic, $T$ and $\Gamma$ are not full. As an example, the $p$-th power (also known as the Frobenius) is a natural transformation

$$
\Phi:\left(V^{\otimes n}\right)_{S_{n}} \longrightarrow\left(V^{\otimes p n}\right)_{S_{p n}},
$$

which is not induced by a morphism of symmetric sequences.

In characteristic 0 , we have a natural isomorphism

$$
\operatorname{Tr}: T \stackrel{\simeq}{\longrightarrow} \Gamma
$$

given by the norm map (cf. 1.1.14). But, in positive characteristic, the functors $T$ and $\Gamma$ are distinct. To be more explicit, as a remark, we mention the following result.

1.1.2. Proposition. Let $M, N$ be symmetric sequences. We assume that the $S_{n^{-}}$ modules $M(n)$ and $N(n)$ are all finite. If there is an isomorphism of functors

$$
\phi: T(M) \stackrel{\simeq}{\longrightarrow} \Gamma(N),
$$

then $M$ and $N$ are necessarily projective. Moreover, there is an isomorphism of symmetric sequences $\phi^{\prime}: M \stackrel{\simeq}{\longrightarrow} N$ such that

$$
\phi=\Gamma\left(\phi^{\prime}\right) \cdot \operatorname{Tr}_{M}=\operatorname{Tr}_{N} \cdot T\left(\phi^{\prime}\right),
$$

where $\operatorname{Tr}: T \longrightarrow \Gamma$ denotes the norm map (cf. 1.1.14). 
A symmetric sequence $M$ is projective if each $S_{n}$-module $M(n)$ is a projective representation.

We do not prove this proposition here; we just mention that the main ingredient of the proof is given by the polynomial filtrations of the functors $T(M)$ and $\Gamma(N)$ (cf. [19]).

1.1.3. The structure of the category of functors on $\mathbf{F}$-modules. Let $S, T \in \mathcal{F}\left(\operatorname{Mod}_{\mathbf{F}}\right)$. The tensor product of $S$ and $T$ is the functor defined by

$$
(S \otimes T)(V)=S(V) \otimes T(V) \text { for } V \in \operatorname{Mod}_{\mathbf{F}} .
$$

The composition product of $S$ and $T$ is the functor defined by

$$
(S \circ T)(V)=S(T(V)) \text { for } V \in \operatorname{Mod}_{\mathbf{F}} .
$$

Clearly, the tensor product of functors

$$
\bigotimes: \mathcal{F}\left(\operatorname{Mod}_{\mathbf{F}}\right) \times \mathcal{F}\left(\operatorname{Mod}_{\mathbf{F}}\right) \longrightarrow \mathcal{F}\left(\operatorname{Mod}_{\mathbf{F}}\right)
$$

is a unital, associative and symmetric bifunctor. Namely, $\left(\mathcal{F}\left(\operatorname{Mod}_{\mathbf{F}}\right), \otimes\right)$ form a symmetric monoidal category (see [23 Chapter XI]). The composition product of functors

$$
\circ: \mathcal{F}\left(\operatorname{Mod}_{\mathbf{F}}\right) \times \mathcal{F}\left(\operatorname{Mod}_{\mathbf{F}}\right) \longrightarrow \mathcal{F}\left(\operatorname{Mod}_{\mathbf{F}}\right)
$$

is a unital and associative (but not symmetric) bifunctor. Namely, $\left(\mathcal{F}\left(\operatorname{Mod}_{\mathbf{F}}\right), \circ\right)$ form a (nonsymmetric) monoidal category (see [23, Chapter VII]). In the sequel, we show that the functors $T: \operatorname{Mod}_{\mathbf{F}}^{S} \longrightarrow \mathcal{F}\left(\operatorname{Mod}_{\mathbf{F}}\right)$ and $\Gamma: \operatorname{Mod}_{\mathbf{F}}^{S} \longrightarrow \mathcal{F}\left(\operatorname{Mod}_{\mathbf{F}}\right)$ reflect the tensor product and the composition product of functors.

1.1.4. The tensor product of symmetric sequences. Let $M$ and $N$ be symmetric sequences. The tensor product of $M$ and $N$ is the symmetric sequence given by the formula

$$
(M \otimes N)(n):=\bigoplus_{i+j=n} \operatorname{Ind}_{S_{i} \times S_{j}}^{S_{n}} M(i) \otimes N(j) .
$$

The tensor product of symmetric sequences

$$
\bigotimes: \operatorname{Mod}_{\mathbf{F}}^{S} \times \operatorname{Mod}_{\mathbf{F}}^{S} \longrightarrow \operatorname{Mod}_{\mathbf{F}}^{S}
$$

is a unital, associative and symmetric bifunctor. Thus, $\left(\operatorname{Mod}_{\mathbf{F}}^{S}, \otimes\right)$ form a symmetric monoidal category (cf. [20, Section 2]). Let us specify the unit and the symmetry isomorphism of $\otimes$. The unit is the symmetric sequence $\mathbf{1}$, which is concentrated in degree 0 , and has $\mathbf{1}(0)=\mathbf{F}$. The symmetry operator

$$
\tau^{*}: M \otimes N \longrightarrow N \otimes M
$$

is characterized as follows. Let $\sigma \in S_{n}, x \in M(i)$ and $y \in N(j)$. Recall that $\sigma \cdot x \otimes y \in(M \otimes N)(n)$ denotes the tensor $\sigma \otimes x \otimes y \in \operatorname{Ind}_{S_{i} \times S_{j}}^{S_{n}} M(i) \otimes N(j)$ (see $0.4)$. We set

$$
\tau^{*}(\sigma \cdot x \otimes y)=\sigma \cdot \tau(i, j) \cdot y \otimes x,
$$

where $\tau(i, j) \in S_{n}$ is the block transposition defined in (0.4). 
1.1.5. Remark. Recall that a symmetric sequence is equivalent to a functor from the category of finite sets and bijections to the category of $\mathbf{F}$-modules (cf. [16], [15]). If $M$ is a symmetric sequence, then the associated functor is given by the formula

$$
M(I):=\mathbf{F}[\mathcal{B} i j(\{1, \ldots, n\}, I)] \otimes_{S_{n}} M(n),
$$

where $n$ is the cardinal of the set $I$. If we start from this numbering-free definition and consider a symmetric sequence as a functor, then the definition of the tensor product of symmetric sequences becomes simpler: we have

$$
(M \otimes N)(I)=\bigoplus_{I_{1} \sqcup I_{2}=I} M\left(I_{1}\right) \otimes N\left(I_{2}\right)
$$

(the sum ranges over the set of ordered partitions of $I$ ). The image of $x \otimes y \in$ $M\left(I_{1}\right) \otimes N\left(I_{2}\right)$ under the symmetry isomorphism is $y \otimes x \in N\left(I_{2}\right) \otimes M\left(I_{1}\right)$ (cf. [20] Section 2]).

1.1.6. Proposition. The functors $T: \operatorname{Mod}_{\boldsymbol{F}}^{S} \longrightarrow \mathcal{F}\left(\operatorname{Mod}_{\mathbf{F}}\right)$ and $\Gamma: \operatorname{Mod}_{\boldsymbol{F}}^{S} \longrightarrow$ $\mathcal{F}\left(\operatorname{Mod}_{\mathbf{F}}\right)$ commute with the tensor product. Explicitly, we have isomorphisms

$$
T(M \otimes N) \simeq T(M) \otimes T(N) \quad \text { and } \quad \Gamma(M \otimes N) \simeq \Gamma(M) \otimes \Gamma(N)
$$

natural in $M, N \in \operatorname{Mod}_{\boldsymbol{F}}^{S}$. Furthermore, these natural isomorphisms preserve the symmetry isomorphisms.

1.1.7. The tensor power of a symmetric sequence. Since the tensor product of symmetric sequences is symmetric, the $r$-th tensor power of a symmetric sequence is equipped with an $S_{r}$-action. Explicitly, let $M$ be a symmetric sequence. Then, any $s \in S_{r}$ yields a morphism of symmetric sequences

$$
s^{*}: M^{\otimes r} \longrightarrow M^{\otimes r} \text {. }
$$

Furthermore, we have $(s t)^{*}=t^{*} s^{*}$. In the sequel, we shall also consider the transformation $s_{*}:=\left(s^{-1}\right)^{*}$.

Let us make the structure of $M^{\otimes r}$ more explicit. We have the identity

$$
M^{\otimes r}(n)=\bigoplus_{\alpha_{1}+\cdots+\alpha_{r}=n} \operatorname{Ind}_{S_{\alpha_{1}} \times \cdots \times S_{\alpha_{r}}}^{S_{n}} M\left(\alpha_{1}\right) \otimes \cdots \otimes M\left(\alpha_{r}\right) .
$$

Let $\sigma \in S_{n}, x_{1} \in M\left(\alpha_{1}\right), \ldots, x_{r} \in M\left(\alpha_{r}\right)$. Recall that $\sigma \cdot x_{1} \otimes \cdots \otimes x_{r} \in M^{\otimes r}(n)$ denotes the tensor

$$
\sigma \otimes x_{1} \otimes \cdots \otimes x_{r} \in \operatorname{Ind}_{S_{\alpha}}^{S_{n}} M\left(\alpha_{1}\right) \otimes \cdots \otimes M\left(\alpha_{r}\right)
$$

(see 0.4). We have

$$
s^{*}\left(\sigma \cdot x_{1} \otimes \cdots \otimes x_{r}\right)=\sigma \cdot s\left(\alpha_{1}, \ldots, \alpha_{r}\right) \cdot x_{s(1)} \otimes \cdots \otimes x_{s(r)},
$$

where $s\left(\alpha_{1}, \ldots, \alpha_{r}\right) \in S_{n}$ is the block permutation defined in (0.4).

1.1.8. The composition product of symmetric sequences. Let $M$ and $N$ be symmetric sequences. The composition product of $M$ and $N$ is the symmetric sequence

$$
M \circ N:=\bigoplus_{r}\left(M(r) \otimes N^{\otimes r}\right)_{S_{r}} .
$$

The composition product of $M$ and $N$ with divided symmetries is the symmetric sequence

$$
M \widetilde{\circ} N:=\bigoplus_{r}\left(M(r) \otimes N^{\otimes r}\right)^{S_{r}} .
$$


The composition product of symmetric sequences and the composition product of symmetric sequences with divided symmetries

$$
\circ: \operatorname{Mod}_{\mathbf{F}}^{S} \times \operatorname{Mod}_{\mathbf{F}}^{S} \longrightarrow \operatorname{Mod}_{\mathbf{F}}^{S} \quad \text { and } \quad \widetilde{\circ}: \operatorname{Mod}_{\mathbf{F}}^{S} \times \operatorname{Mod}_{\mathbf{F}}^{S} \longrightarrow \operatorname{Mod}_{\mathbf{F}}^{S}
$$

are both unital and associative (but not symmetric) bifunctors. In fact, the symmetric sequence $I$, which is concentrated in degree 1 and has $I(1)=\mathbf{F}$, is a unit for both of these composition products.

1.1.9. Proposition. The functors $T: \operatorname{Mod}_{\boldsymbol{F}}^{S} \longrightarrow \mathcal{F}\left(\operatorname{Mod}_{\mathbf{F}}\right)$ and $\Gamma: \operatorname{Mod}_{\boldsymbol{F}}^{S} \longrightarrow$ $\mathcal{F}\left(\operatorname{Mod}_{\mathbf{F}}\right)$ reflect the composition product of functors. More precisely, we have isomorphisms

$$
T(M \circ N) \simeq T(M) \circ T(N) \quad \text { and } \quad \Gamma(M \widetilde{\circ} N) \simeq \Gamma(M) \circ \Gamma(N)
$$

natural in $M, N \in \operatorname{Mod}_{F}^{S}$.

1.1.10. Operads. An operad $\mathcal{P}$ is a symmetric sequence equipped with an associative product $\mu: \mathcal{P} \circ \mathcal{P} \longrightarrow \mathcal{P}$ together with a unit $\eta: I \longrightarrow \mathcal{P}$ (cf. [15]). By construction of the composition product of symmetric sequences, it is equivalent to give products

$$
\mathcal{P}(n) \otimes \mathcal{P}\left(\alpha_{1}\right) \otimes \cdots \otimes \mathcal{P}\left(\alpha_{n}\right) \longrightarrow \mathcal{P}\left(\alpha_{1}+\cdots+\alpha_{n}\right),
$$

which are associative, unital, and equivariant in some natural sense (cf. [26, Chapter $1])$. The image of $p \otimes q_{1} \otimes \cdots \otimes q_{n} \in \mathcal{P}(n) \otimes \mathcal{P}\left(\alpha_{1}\right) \otimes \cdots \otimes \mathcal{P}\left(\alpha_{n}\right)$ under the operad product is denoted by $p\left(q_{1}, \ldots, q_{n}\right)$. The element $1 \in \mathcal{P}(1)$ represents the unit of the operad.

1.1.11. Algebras over an operad. By the last proposition, $\mu$ induces a unital and associative product $T(\mathcal{P}) \circ T(\mathcal{P}) \longrightarrow T(\mathcal{P})$. Hence, $T(\mathcal{P})$ is equipped with a monad structure (cf. 26, Chapter 2]). An algebra over $T(\mathcal{P})$, also known as a $\mathcal{P}$-algebra, is an $\mathbf{F}$-module $A$ together with a product $\lambda: T(\mathcal{P}, A) \longrightarrow A$, which is associative and unital with respect to the monad structure. It is equivalent to equip $A$ with $S_{n}$-invariant products

$$
\mathcal{P}(n) \otimes A^{\otimes n} \longrightarrow A
$$

which are associative and unital with respect to the operad structure. The image of $p \otimes a_{1} \otimes \cdots \otimes a_{n} \in \mathcal{P}(n) \otimes A^{\otimes n}$ under the $\mathcal{P}$-algebra product is denoted by $p\left(a_{1}, \ldots, a_{n}\right)$. By definition, $T(\mathcal{P}, V)$ is the free $\mathcal{P}$-algebra generated by $V$. Recall that we can extend the functor $T(\mathcal{P},-)$ to the category of graded $\mathbf{F}$-modules. Therefore, we have a notion of a graded $\mathcal{P}$-algebra.

1.1.12. Connected operads and augmented $\mathcal{P}$-algebras. Recall that an operad $\mathcal{P}$ is connected if $\mathcal{P}(0)=0$. If $\mathcal{P}$ is not connected, then let $K=\mathcal{P}(0)$. Furthermore, let $\overline{\mathcal{P}}$ be the connected operad such that

$$
\overline{\mathcal{P}}(n)= \begin{cases}0, & \text { if } n=0 \\ \mathcal{P}(n), & \text { otherwise }\end{cases}
$$

Clearly, $K$ is a $\overline{\mathcal{P}}$-algebra. Moreover, the structure of a $\mathcal{P}$-algebra is equivalent to the structure of a $\overline{\mathcal{P}}$-algebra under $K$. Recall that a $\overline{\mathcal{P}}$-algebra under $K$ is a $\overline{\mathcal{P}}$ algebra $A$ equipped with a fixed $\overline{\mathcal{P}}$-algebra morphism $\eta_{A}: K \longrightarrow A$. Conversely, a $\overline{\mathcal{P}}$-algebra is equivalent to a $\mathcal{P}$-algebra over $K$. Recall that a $\mathcal{P}$-algebra over $K$ is a $\mathcal{P}$-algebra $A$ equipped with a fixed $\mathcal{P}$-algebra morphism $\epsilon_{A}: A \longrightarrow K$. This morphism $\epsilon_{A}$ is also known as an augmentation, and a $\mathcal{P}$-algebra over $K$ is also known as an augmented $\mathcal{P}$-algebra. 
In the sequel, if $\mathcal{P}$ is an operad, then we tacitly assume that $\mathcal{P}$ is connected. We concentrate on connected operads, because the divided power structure of a commutative algebra $A$ is carried by the augmentation ideal of $A$ (see 1.2.2). Furthermore, the connectedness hypothesis is required to equip the functor $\Gamma(\mathcal{P},-)$ with a monad structure (see proposition 1.1.15), and hence, to generalize the notion of a divided power algebra.

1.1.13. Examples. As mentioned in the introduction, there are operads $\mathcal{C}$ om, $\mathcal{A} s$, $\mathcal{L}$ ie and $\mathcal{P}$ ois, whose algebras are, respectively, commutative algebras, associative algebras, Lie algebras and Poisson algebras. We follow the notation of Ginzburg and Kapranov (cf. [16]). Thus, the operads $\mathcal{C}$ om, $\mathcal{A} s, \mathcal{L}$ ie and $\mathcal{P}$ ois are connected and their algebras have no unit. We denote by $\mathcal{C o m}_{+}, \mathcal{A} s_{+}$and $\mathcal{P}$ ois $s_{+}$the associated operads for unital algebras. If $\mathcal{P}=\mathcal{C}$ om, $\mathcal{A} s$ or $\mathcal{P}$ ois, then we have

$$
\mathcal{P}_{+}(n)= \begin{cases}\mathbf{F}, & \text { if } n=0, \\ \mathcal{P}(n), & \text { otherwise. }\end{cases}
$$

In characteristic 2, a Lie bracket is just assumed to be antisymmetric. We do not have necessarily $[x, x]=0$. In fact, we cannot encode such a relation in the structure of an algebra over an operad, because this relation is not linear. Recall that the free associative algebra is the tensor algebra

$$
T(\mathcal{A} s, V)=\bar{T}(V)=\bigoplus_{n \geq 1} V^{\otimes n}
$$

and the free commutative algebra is the symmetric algebra

$$
T(\mathcal{C o m}, V)=\bar{S}(V)=\bigoplus_{n \geq 1}\left(V^{\otimes n}\right)_{S_{n}} .
$$

The free Lie algebra is more difficult to describe (see [27]). The Poisson operad is the composite $\mathcal{P}$ ois $=\mathcal{C}$ om $\circ \mathcal{L}$ ie (cf. 1.2.17). As a consequence, we have

$$
T(\mathcal{P} o i s, V)=T(\mathcal{C o m}, T(\mathcal{L} i e, V)) \text {. }
$$

An element of the $S_{n}$-module $\mathcal{P}(n)$ can be regarded as a multilinear polynomial in $n$-variables $X_{1}, \ldots, X_{n}$ for the algebra structures associated to $\mathcal{P}$. The symmetric group $S_{n}$ acts on $\mathcal{P}(n)$ by permutation of the variables. For example, we have

$$
\begin{aligned}
\mathcal{A} s(n) & =\operatorname{Span}\left\langle X_{s(1)} \cdots X_{s(n)} ; s \in S_{n}\right\rangle, \\
\mathcal{C} o m(n) & =\operatorname{Span}\left\langle X_{1} \cdots X_{n}\right\rangle, \\
\mathcal{L} i e(n) & =\operatorname{Span}\left\langle\left[\left[\cdots\left[X_{s(1)}, X_{s(2)}\right], \ldots\right], X_{s(n)}\right] ; s \in S_{n} \text { and } s(1)=1\right\rangle .
\end{aligned}
$$

We use this representation in the calculations of the next section.

To complete this section, we compare the functors $T$ and $\Gamma$. In particular, if $\mathcal{P}$ is a connected operad, then we show that $\Gamma(\mathcal{P})$ is equipped with a monad structure.

1.1.14. The norm map. Let $X$ be an $S_{r}$-module. The norm map is the natural transformation

$$
\operatorname{Tr}: X_{S_{r}} \longrightarrow X^{S_{r}}
$$

given by $\operatorname{Tr}(x)=\sum_{s \in S_{r}} s x$.

For $M$ a symmetric sequence and $V$ an $\mathbf{F}$-module, we have a natural transformation

$$
\operatorname{Tr}_{M, V}: T(M, V) \longrightarrow \Gamma(M, V)
$$


given by the norm map above. Similarly, if $M$ and $N$ are symmetric sequences, then we have a morphism of symmetric sequences

$$
\operatorname{Tr}_{M, N}: M \circ N \longrightarrow M \widetilde{\circ} N
$$

In general, the norm map $\operatorname{Tr}: X_{S_{r}} \longrightarrow X^{S_{r}}$ is not an isomorphism. In particular, $\operatorname{Tr}_{M, V}: T(M, V) \longrightarrow \Gamma(M, V)$ is not an isomorphism of functors. Nevertheless, for symmetric sequences, we have

1.1.15. Proposition. Let $M, N$ be symmetric sequences. If $N$ is connected, then the norm map

$$
\operatorname{Tr}_{M, N}: M \circ N \longrightarrow M \widetilde{\circ} N
$$

is an isomorphism. (Recall that $N$ is connected if $N(0)=0$.)

This proposition is an immediate consequence of the following lemma.

1.1.16. Lemma. If $N$ is connected, then $S_{r}$ acts freely on the symmetric sequence $N^{\otimes r}$. More precisely, there are $S_{n}$-modules $G(n), n \geq 0$, such that we have $S_{r}-S_{n}$ bimodule identities

$$
N^{\otimes r}(n)=\mathbf{F}\left[S_{r}\right] \otimes G(n), n \geq 0 .
$$

The lemma is immediate, if one calls for the equivalent definition of the tensor product of symmetric sequences, which is given in remark 1.1.5. Nevertheless, we give another proof because we need insights into the proposition.

Fix an integer $n$. Below, we define a groupoid $G_{\alpha}$, whose objects are indexed by the set of $r$-tuples of positive integers $\alpha=\left(\alpha_{1}, \ldots, \alpha_{r}\right)$ such that $\alpha_{1}+\cdots+\alpha_{r}=n$. The groups $S_{r}$ and $S_{\alpha}$ embed into the morphisms of $G_{\alpha}$. Furthermore, we have an $S_{r}-S_{n}$-module identity

$$
N^{\otimes r}(n)=\bigoplus_{\alpha} \mathbf{F}\left[G_{\alpha}\right] \otimes_{S_{\alpha}}\left(N\left(\alpha_{1}\right) \otimes \cdots \otimes N\left(\alpha_{r}\right)\right) .
$$

The lemma is an immediate consequence of the fact 1.1.17 below.

The object indexed by $\alpha$ is set to be the set $G_{\alpha}=S_{n}$. The morphisms of the groupoid are the composites of the following bijections:

- Let $\sigma_{i} \in S_{\alpha_{i}}, i=1, \ldots, r$. The right translate by $\sigma_{1} \oplus \cdots \oplus \sigma_{r}$ gives rise to

$$
\left(\sigma_{1} \oplus \cdots \oplus \sigma_{r}\right)^{*}: G_{\alpha} \longrightarrow G_{\alpha} .
$$

- Let $s \in S_{r}$. We denote by $\alpha^{s}$ the $r$-tuple $\left(\alpha_{s(1)}, \ldots, \alpha_{s(r)}\right)$. The right translate by $s\left(\alpha_{1}, \ldots, \alpha_{r}\right)$ gives rise to

$$
s^{*}: G_{\alpha} \longrightarrow G_{\alpha^{s}} .
$$

If $\sigma_{i}, \tau_{i} \in S_{\alpha_{i}}, i=1, \ldots, r$, then we have

$$
\left(\sigma_{1} \oplus \cdots \oplus \sigma_{r}\right)^{*}\left(\tau_{1} \oplus \cdots \oplus \tau_{r}\right)^{*}=\left(\tau_{1} \sigma_{1} \oplus \cdots \oplus \tau_{r} \sigma_{r}\right)^{*} .
$$

If $s, t \in S_{r}$, then $t^{*} s^{*}=(s t)^{*}$. Moreover, for $s, \sigma_{1}, \ldots, \sigma_{r}$ as above, we have

$$
\left(\sigma_{s(1)} \oplus \cdots \oplus \sigma_{s(r)}\right)^{*} s^{*}=s^{*}\left(\sigma_{1} \oplus \cdots \oplus \sigma_{r}\right)^{*} .
$$

Thus, any morphism $u: G_{\alpha} \longrightarrow G_{\beta}$ has a decomposition of the form

$$
u=s^{*}\left(\sigma_{1} \oplus \cdots \oplus \sigma_{r}\right)^{*} \text {. }
$$

The following fact implies that such a decomposition is unique.

1.1.17. Fact. Let $s, \sigma_{1}, \ldots, \sigma_{r}$ be as above. If $s^{*}\left(\sigma_{1} \oplus \cdots \oplus \sigma_{r}\right)^{*} w=w$ for some $w \in G_{\alpha}$, then $s=1$, and $\sigma_{1}=1, \ldots, \sigma_{r}=1$. 
1.1.18. Algebras with divided symmetries. Now, if $\mathcal{P}$ is a connected operad, then there is a product: $\tilde{\mu}: \mathcal{P} \widetilde{\circ} \longrightarrow \mathcal{P}$. If $\mu: \mathcal{P} \circ \mathcal{P} \longrightarrow \mathcal{P}$ is the operad product, then $\widetilde{\mu}$ is the composite

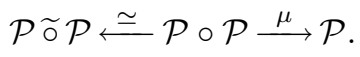

Observe that $\widetilde{\mu}$ is unital and associative, because $\mu$ is, and because the norm map $\operatorname{Tr}_{M, N}$ is "associative" in an appropriate sense. As a consequence, the functor $\Gamma(\mathcal{P})$ is equipped with a monad structure. Let us recapitulate the definition of the monad product

$$
\Gamma(\mathcal{P}, \Gamma(\mathcal{P}, V)) \longrightarrow \Gamma(\mathcal{P}, V)
$$

We compose the following morphisms. First, we interchange $\Gamma$ with the tensor power

$$
\bigoplus_{r}\left(\mathcal{P}(r) \otimes \Gamma(\mathcal{P}, V)^{\otimes r}\right)^{S_{r}} \stackrel{\simeq}{\longrightarrow} \bigoplus_{r}\left(\mathcal{P}(r) \otimes \Gamma\left(\mathcal{P}^{\otimes r}, V\right)\right)^{S_{r}}
$$

Then, we have the identities

$$
\begin{aligned}
& \bigoplus_{r}\left(\mathcal{P}(r) \otimes\left(\mathcal{P}^{\otimes r}(n) \otimes V^{\otimes n}\right)^{S_{n}}\right)^{S_{r}} \\
& =\bigoplus_{r}\left(\left(\mathcal{P}(r) \otimes \mathcal{P}^{\otimes r}\right)^{S_{r}}(n) \otimes V^{\otimes n}\right)^{S_{n}}
\end{aligned}
$$

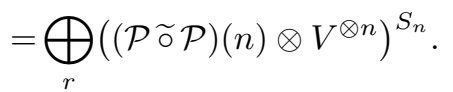

These operations compose the canonical isomorphism

$$
\Gamma(\mathcal{P}, \Gamma(\mathcal{P}, V)) \stackrel{\simeq}{\longrightarrow} \Gamma(\mathcal{P} \widetilde{\circ} \mathcal{P}, V)
$$

We compose this isomorphism with the inverse of the norm map

$$
\Gamma(\mathcal{P} \widetilde{\mathcal{P}}, V) \stackrel{\simeq}{\longleftarrow}(\mathcal{P} \circ \mathcal{P}, V)
$$

Finally, we apply the operad product

$$
\Gamma(\mathcal{P} \circ \mathcal{P}, V) \longrightarrow \Gamma(\mathcal{P}, V) .
$$

We define a $\mathcal{P}$-algebra with divided symmetries (a $\Gamma \mathcal{P}$-algebra for short) to be an algebra over the monad $\Gamma(\mathcal{P})$. In general, $T(\mathcal{P})$ and $\Gamma(\mathcal{P})$ are distinct functors. Therefore, the $\Gamma \mathcal{P}$-algebras and the $\mathcal{P}$-algebras are not equivalent structures.

Nevertheless, the next lemma implies that the norm map

$$
\operatorname{Tr}: T(\mathcal{P}, V) \longrightarrow \Gamma(\mathcal{P}, V)
$$

is a morphism of monads. Because of this property, we have a forgetful functor from the category of $\Gamma \mathcal{P}$-algebras to the category of $\mathcal{P}$-algebras. Explicitly, if $A$ is a $\Gamma \mathcal{P}$-algebra, then the composite of the norm map with the $\Gamma \mathcal{P}$-algebra product

$$
T(\mathcal{P}, A) \stackrel{\operatorname{Tr}}{\longrightarrow} \Gamma(\mathcal{P}, A) \longrightarrow A
$$

equips $A$ with a $\mathcal{P}$-algebra product. 
1.1.19. Lemma. The obvious diagram

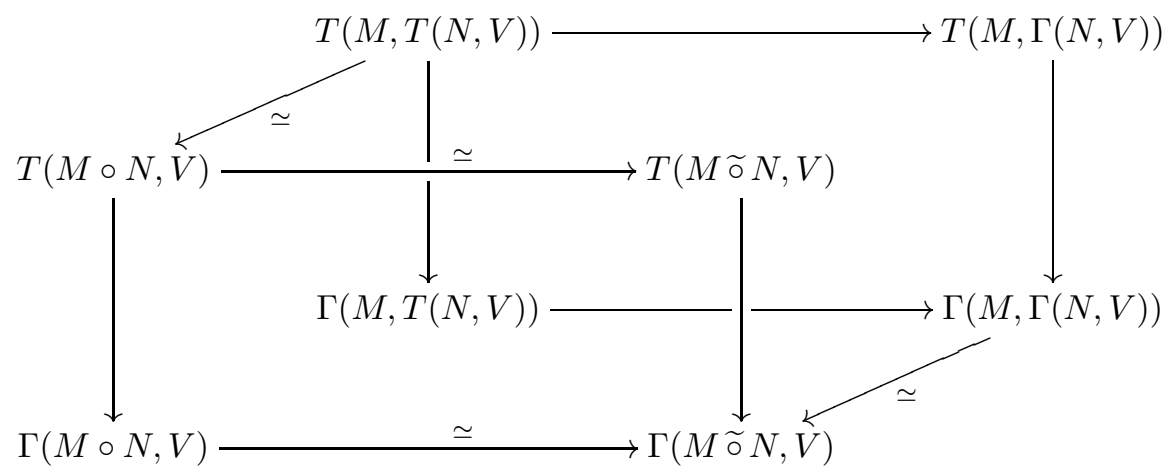

is commutative.

Proof. The squares in the foreground and in the background commute by naturality of the norm map. The commutativity of the whole diagram follows from a straightforward verification.

1.1.20. The $\mathcal{P}$-algebra structure of the free $\Gamma \mathcal{P}$-algebra. As an example, the free $\Gamma \mathcal{P}$ algebra $A=\Gamma(\mathcal{P}, V)$ is endowed with a $\mathcal{P}$-algebra structure. The $\mathcal{P}$-algebra product $T(\mathcal{P}, \Gamma(\mathcal{P}, V)) \longrightarrow \Gamma(\mathcal{P}, V)$ is the composite of the norm map $T(\mathcal{P}, \Gamma(\mathcal{P}, V)) \longrightarrow$ $\Gamma(\mathcal{P}, \Gamma(\mathcal{P}, V))$ with the monad product $\Gamma(\mathcal{P}, \Gamma(\mathcal{P}, V)) \longrightarrow \Gamma(\mathcal{P}, V)$. The lemma implies also that the norm map $T(\mathcal{P}, V) \longrightarrow \Gamma(\mathcal{P}, V)$ is a $\mathcal{P}$-algebra morphism.

Let $p \in \mathcal{P}(r), \gamma_{1}, \ldots, \gamma_{r} \in \Gamma(\mathcal{P}, V)$. We write down an explicit formula for the product $p\left(\gamma_{1}, \ldots, \gamma_{r}\right)$ in $\Gamma(\mathcal{P}, V)$. Assume that $\gamma_{k}$ is the tensor

$$
\sum_{i} q_{(k)}^{i} \otimes \underline{v}_{(k)}^{i} \in\left(\mathcal{P}\left(\alpha_{k}\right) \otimes V^{\otimes \alpha_{k}}\right)^{S_{\alpha_{k}}}
$$

where $q_{(k)}^{i} \in \mathcal{P}\left(\alpha_{k}\right)$ and $\underline{v}_{(k)}^{i} \in V^{\otimes \alpha_{k}}$. Let $n=\alpha_{1}+\cdots+\alpha_{r}$. By concatenation, we obtain the word

$$
\underline{v}_{(1)}^{i_{1}} \cdots \underline{v}_{(r)}^{i_{r}} \in V^{\otimes n}
$$

and if $s \in S_{n}$, then by place permutation we obtain the word denoted by

$$
s_{*}\left(\underline{v}_{(1)}^{i_{1}} \cdots \underline{v}_{(r)}^{i_{r}}\right) \in V^{\otimes n} .
$$

We have

$$
p\left(\gamma_{1}, \ldots, \gamma_{r}\right)=\sum_{s \in S_{n} / S_{\alpha}}\left(s \cdot p\left(q_{(1)}^{i_{1}}, \ldots, q_{(r)}^{i_{r}}\right)\right) \otimes s_{*}\left(\underline{(}_{(1)}^{i_{1}} \cdots \underline{v}_{(r)}^{i_{r}}\right) .
$$

1.2. Examples. In this section, we give an explicit description of the structure of a $\Gamma \mathcal{P}$-algebra for the classical operads $\mathcal{P}=\mathcal{C}$ om, $\mathcal{A} s, \mathcal{L}$ ie, Pois. In each case, a $\Gamma \mathcal{P}$-algebra is just a $\mathcal{P}$-algebra equipped with additional operations.

1.2.1. The case of a projective operad. Let $\mathcal{P}$ be an operad. If $\mathcal{P}$ is projective as a symmetric sequence (for example, if $\mathcal{P}=\mathcal{A} s$ ), then the norm map $\operatorname{Tr}: T(\mathcal{P}, V) \longrightarrow$ $\Gamma(\mathcal{P}, V)$ is an isomorphism. In other words, in this case, a $\Gamma \mathcal{P}$-algebra does not carry more structure than a $\mathcal{P}$-algebra. 
1.2.2. Divided power algebras. Let us recall the notion of a divided power algebra. Let $A$ be an augmented (graded) commutative algebra. Let $\bar{A}$ denote the augmentation ideal of $A$. The unital algebra $A$ (or the nonunital algebra $\bar{A}$ ) is equipped with the structure of a divided power algebra if there are operations $\gamma_{i}: \bar{A} \longrightarrow \bar{A}$, $i>0$, such that

$$
\begin{gathered}
\gamma_{1}(x)=x, \quad \gamma_{i}(x+y)=\sum_{j=0}^{i} \gamma_{j}(x) \gamma_{i-j}(y), \\
\gamma_{i}(x y)=i ! \gamma_{i}(x) \gamma_{i}(y), \quad \gamma_{i}(x) \gamma_{j}(x)=\frac{(i+j) !}{i ! j !} \gamma_{i+j}(x),
\end{gathered}
$$

and

$$
\gamma_{i}\left(\gamma_{j}(x)\right)=\frac{(i j) !}{i !(j !)^{i}} \gamma_{i j}(x)
$$

(By convention $\gamma_{0}(x):=1, \forall x \in \bar{A}$.) In the graded case, the divided powers $\gamma_{i}$ are supposed to multiply the degree by $i$ and in the case $p \neq 2$ to vanish in odd degree.

The next proposition is well known (cf. [5], [28]).

1.2.3. Proposition. The free nonunital (graded) divided power algebra functor is given by

$$
\Gamma(\mathcal{C o m}, V)=\bigoplus_{n \geq 1}\left(V^{\otimes n}\right)^{S_{n}} .
$$

As a consequence, a (graded) $\Gamma \mathcal{C}$ om-algebra is nothing but a (graded) divided power algebra. Let $A$ be a $\Gamma \mathcal{C}$ om-algebra. The $i$-th divided power $\gamma_{i}(a)$ of $a \in A$ is the image of $a^{\otimes i} \in \Gamma(\mathcal{C}$ om,$A)$ under the $\Gamma \mathcal{C}$ om-algebra product $\lambda: \Gamma(\mathcal{C}$ om,$A) \longrightarrow$ A.

1.2.4. Restricted Lie algebras. Recall that a (graded) restricted Lie algebra is a (graded) Lie algebra $g$ equipped with a Frobenius $F: g \longrightarrow g$. In the graded case, $F$ is supposed to multiply the degree by $p$ and to vanish in odd degree in the case $p \neq 2$. We denote by $\mathcal{L}_{p}(V)$ the free (graded) restricted Lie algebra generated by V.

Recall that $\mathcal{A} s_{+}$denotes the operad of unital associative algebras (cf. 1.1.13). We have

$$
\mathcal{A} s_{+}(n)= \begin{cases}\mathbf{F}, & \text { if } n=0 \\ \mathcal{A} s(n), & \text { otherwise }\end{cases}
$$

Thus,

$$
T\left(\mathcal{A} s_{+}, V\right)=T(V)=\bigoplus_{n \geq 0} V^{\otimes n} .
$$

Furthermore, observe that $\Gamma\left(\mathcal{A} s_{+}, V\right)=T\left(\mathcal{A} s_{+}, V\right)=T(V)$. As a consequence, $\Gamma\left(\mathcal{A} s_{+},-\right)$is equipped with a monad structure, despite the fact that $\mathcal{A} s_{+}$is nonconnected. The operad embedding $\mathcal{L} i e \longleftrightarrow \mathcal{A} s_{+}$induces a monad embedding $\Gamma(\mathcal{L} i e, V) \longleftrightarrow \Gamma\left(\mathcal{A} s_{+}, V\right)=T(V)$.

On the other hand, any unital (graded) associative algebra $A$ has a structure of a restricted Lie algebra; the Lie bracket is given by $[a, b]:=a b-b a, \forall a, b \in A$, and the Frobenius by $F(a):=a^{p}, \forall a \in A$. As a consequence, we have a morphism of monads $\mathcal{L}_{p}(V) \longrightarrow T(V)$. Furthermore, it is well known that this morphism is an embedding (cf. [21, Section V.7]). 
1.2.5. Theorem. The sub-monads $\Gamma(\mathcal{L} i e, V) \subseteq T(V)$ and $\mathcal{L}_{p}(V) \subseteq T(V)$ are equal. A (graded) $\Gamma \mathcal{L}$ ie-algebra is, in fact, a (graded) restricted Lie algebra.

For simplicity, we work with ungraded objects. First, we prove that $\Gamma(\mathcal{L} i e, V)$ is stable under the Lie bracket and the Frobenius in $T(V)$. These assertions follow respectively from the lemma 1.2 .6 and from the lemma 1.2.7 below. As a consequence, we obtain $\mathcal{L}_{p}(V) \subseteq \Gamma(\mathcal{L} i e, V)$. The tensor algebra $T(V)$ is equipped with a coproduct known as the shuffle coproduct. Furthermore, it is known that $\mathcal{L}_{p}(V) \subseteq T(V)$ is the primitive part of $T(V)$ (cf. [21, Section V.7]). We prove that $\Gamma(\mathcal{L} i e, V)$ is primitive in $T(V)$. Hence, finally, we also have the inclusion $\Gamma(\mathcal{L} i e, V) \subseteq \operatorname{Prim} T(V)=\mathcal{L}_{p}(V)$, and the theorem follows. Let us mention that the second assertion of the theorem is an immediate consequence of the first one.

1.2.6. Lemma. Let $V$ be the $\mathbf{F}$-module generated by $x$ and $y$. We have $[x, y] \in$ $\Gamma(\mathcal{L} i e, V)$.

Proof. The tensors $X_{1} \otimes x, X_{1} \otimes y \in\left(\mathcal{A} s_{+}(1) \otimes V\right)^{S_{1}}$ are the images of $x, y \in V$ under the canonical embedding $V \longrightarrow \Gamma\left(\mathcal{A} s_{+}, V\right)$. By (1.1.20), the bracket $[x, y]=$ $x y-y x$ in $\Gamma\left(\mathcal{A} s_{+}, V\right)$ is given by the formula

$$
X_{1} X_{2} \otimes(x \otimes y)+X_{2} X_{1} \otimes(y \otimes x)-X_{1} X_{2} \otimes(y \otimes x)-X_{2} X_{1} \otimes(x \otimes y) .
$$

This last expression is equal to

$$
\left[X_{1}, X_{2}\right] \otimes(x \otimes y)+\left[X_{2}, X_{1}\right] \otimes(y \otimes x),
$$

which belongs to $\left(\mathcal{L} i e(2) \otimes V^{\otimes 2}\right)^{S_{2}} \subseteq \Gamma(\mathcal{L} i e, V)$.

1.2.7. Lemma. Let $V$ be the $\mathbf{F}$-module generated by $x$. We have $x^{p} \in \Gamma(\mathcal{L} i e, V)$.

Proof. By (1.1.20), the $p$-th power of $x$ in $\Gamma\left(\mathcal{A} s_{+}, V\right)$ is given by the formula

$$
\sum_{s \in S_{p}} X_{s(1)} \cdots X_{s(p)} \otimes x^{\otimes p}
$$

Thus, we have to show that $\sum_{s \in S_{p}} X_{s(1)} \cdots X_{s(p)}$ is a Lie polynomial. This follows from the Jacobson relation: If $X$ and $Y$ are noncommutative variables, then $(X+Y)^{p}-X^{p}-Y^{p}$ is a sum of Lie polynomials (cf. 21. Section V.7]). By an immediate induction, the product $\sum_{s \in S_{p}} X_{s(1)} \cdots X_{s(p)}$, which is the multilinear part of $\left(X_{1}+\cdots+X_{p}\right)^{p}-X_{1}^{p}-\cdots-X^{p}$, is a Lie polynomial.

1.2.8. Remark. It is possible to write down an explicit formula for the Frobenius. In fact, after an elementary enumeration exercise, one may show the identity

$$
\sum_{s \in S_{p}} X_{s(1)} \cdots X_{s(p)}=\sum_{s(1)=1}\left[\left[\cdots\left[X_{s(1)}, X_{s(2)}\right], \ldots\right], X_{s(p)}\right] .
$$

Hence, the image of $x \in V$ under the Frobenius in $\Gamma(\mathcal{L} i e, V)$ is given by the formula

$$
F(x)=\left(\sum_{s(1)=1}\left[\left[\cdots\left[X_{s(1)}, X_{s(2)}\right], \ldots\right], X_{s(p)}\right]\right) \otimes x^{\otimes p} .
$$

1.2.9. Remark. Notice that if $g$ is a 2-restricted Lie algebra (in our sense), then we have $[x, x]=2 F(x)=0, \forall x \in g$.

Now, we prove that $\Gamma(\mathcal{L} i e, V)$ is primitive in $T(V)$. For this purpose, we introduce the following notion: 
1.2.10. Hopf algebras in the monoidal category of symmetric sequences. One may define the structure of an associative algebra, the structure of a cocommutative algebra and the structure of a Hopf algebra in any symmetric monoidal category (see [23, 26, Chapter VII], [14 Appendix A]). Explicitly, let $\mathcal{M}$ be a symmetric monoidal category. We denote the tensor product of $\mathcal{M}$ by $\otimes$ and the unit of $\otimes$ by 1. An associative algebra in $\mathcal{M}$ is an object $A \in \mathcal{M}$ equipped with a unit $\eta: \mathbf{1} \longrightarrow A$ and a product $\mu: A \otimes A \longrightarrow A$. Moreover, the product $\mu$ is assumed to be associative and has a unit given by $\eta$. More explicitly, we have the classical relations:

$$
\mu \cdot \mu \otimes 1_{A}=\mu \cdot 1_{A} \otimes \mu \quad \text { and } \quad \mu \cdot \eta \otimes 1_{A}=1_{A}=\mu \cdot 1_{A} \otimes \eta .
$$

Similarly, a cocommutative coalgebra in $\mathcal{M}$ is an object $C \in \mathcal{M}$ equipped with an augmented coassociative and cocommutative coproduct $\Delta: C \longrightarrow C \otimes C$. The augmentation is a morphism $\epsilon: C \longrightarrow \mathbf{1}$. Thus, we have

$$
\Delta \otimes 1_{C} \cdot \Delta=1_{C} \otimes \Delta \cdot \Delta, \quad \tau^{*} \cdot \Delta=\Delta
$$

and

$$
\epsilon \otimes 1_{C} \cdot \Delta=1_{C}=1_{C} \otimes \epsilon \cdot \Delta
$$

A cocommutative Hopf algebra in $\mathcal{M}$ is an object $H \in \mathcal{M}$ equipped with both a structure of an associative algebra and a structure of a cocommutative coalgebra. Furthermore, as for classical Hopf algebras, these structures are supposed to be compatible. Explicitly, we have

$$
\Delta \cdot \mu=\mu \otimes \mu \cdot 1_{H} \otimes \tau^{*} \otimes 1_{H} \cdot \Delta \otimes \Delta .
$$

We consider a Hopf algebra $H$ in the category of symmetric sequences equipped with the tensor product of symmetric sequences. We say that $H$ is a connected Hopf algebra if $H(0)=\mathbf{F}$. In this case, the unit and the augmentation of $H$ are the identity on $H(0)=\mathbf{1}(0)$. (Recall that the unit for the tensor product of symmetric sequences is concentrated in degree 0 and has $\mathbf{1}(0)=\mathbf{F}$.)

The functors $T: \operatorname{Mod}_{\mathbf{F}}^{S} \longrightarrow \mathcal{F}\left(\operatorname{Mod}_{\mathbf{F}}\right)$ and $\Gamma: \operatorname{Mod}_{\mathbf{F}}^{S} \longrightarrow \mathcal{F}\left(\operatorname{Mod}_{\mathbf{F}}\right)$ preserve the tensor product. Therefore, if $H$ is a Hopf algebra in $\left(\operatorname{Mod}_{\mathbf{F}}^{S}, \otimes\right)$, then $T(H)$ and $\Gamma(H)$ are Hopf algebras in $\left(\mathcal{F}\left(\operatorname{Mod}_{\mathbf{F}}\right), \otimes\right)$. Equivalently, by definition of the tensor product of functors, the $\mathbf{F}$-modules $T(H, V)$ and $\Gamma(H, V)$ are equipped with a structure of a (cocommutative) Hopf algebra which is natural in $V \in \operatorname{Mod}_{\mathbf{F}}$. Explicitly, the product and the coproduct of $T(H, V)$ are the composite:

$$
T(H, V) \otimes T(H, V) \simeq T(H, V) \stackrel{T(\mu, V)}{\longrightarrow} T(H, V)
$$

and

$$
T(H, V) \underset{T(\Delta, V)}{\longrightarrow} T(H, V) \simeq T(H, V) \otimes T(H, V)
$$

If $H$ is a connected Hopf algebra, then the unit and the augmentation of $T(H, V)$ are given by the identification of $\mathbf{F}$ with the degree 0 component of $T(H, V)$. The Hopf algebra structure of $\Gamma(H, V)$ is similar.

1.2.11. The Hopf algebra structure of the associative operad $\mathcal{A} s_{+}$. We have mentioned that $T(V)$ is equipped with a structure of a cocommutative Hopf algebra. 
The coproduct of $\left(v_{1}, \ldots, v_{n}\right) \in T(V)$ (known as the shuffle coproduct) is given by the formula:

$$
\Delta(v)=\sum_{i+j=n}\left\{\sum_{s}\left(v_{s(1)}, \ldots, v_{s(i)}\right) \otimes\left(v_{s(i+1)}, \ldots, v_{s(i+j)}\right)\right\} .
$$

(The sum inside the brackets ranges over all $(i, j)$-shuffles.)

We claim that this Hopf algebra structure is induced by a Hopf algebra structure on the symmetric sequence $\mathcal{A} s_{+}$. Let $P_{s} \in \mathcal{A} s_{+}(n), s \in S_{n}$, denote the monomial $X_{s(1)} \cdots X_{s(n)}$. We have the identity $s \cdot P_{t}=P_{s t}, s, t \in S_{n}$. In this paragraph, we denote also the unit of $S_{n}$ by $1_{n}$ in order to distinguish between them. Recall that

$$
\left(\mathcal{A} s_{+} \otimes \mathcal{A} s_{+}\right)(n)=\bigoplus_{i+j=n} \operatorname{Ind}_{S_{i} \times S_{j}}^{S_{n}} \mathcal{A} s_{+}(i) \otimes \mathcal{A} s_{+}(j) .
$$

The product $\mu: \mathcal{A} s_{+} \otimes \mathcal{A} s_{+} \longrightarrow \mathcal{A} s_{+}$is characterized by

$$
\mu\left(P_{s} \otimes P_{t}\right)=P_{s \oplus t},
$$

for $P_{s} \in \mathcal{A} s_{+}(i), P_{t} \in \mathcal{A} s_{+}(j)$. Equivalently, $\mu\left(P_{s} \otimes P_{t}\right)$ is the image of $X_{1} X_{2} \otimes$ $P_{s} \otimes P_{t}$ under the operad product

$$
\mathcal{A} s_{+}(2) \otimes \mathcal{A} s_{+}(i) \otimes \mathcal{A} s_{+}(j) \longrightarrow \mathcal{A} s_{+}(i) \otimes \mathcal{A} s_{+}(j) .
$$

By equivariance, for a tensor $\sigma \cdot P_{s} \otimes P_{t} \in \operatorname{Ind}_{S_{i} \times S_{j}}^{S_{n}} \mathcal{A} s_{+}(i) \otimes \mathcal{A} s_{+}(j)$, we obtain

$$
\mu\left(\sigma \cdot P_{s} \otimes P_{t}\right)=\sigma \cdot P_{s \oplus t} .
$$

The coproduct $\Delta: \mathcal{A} s_{+} \longrightarrow \mathcal{A} s_{+} \otimes \mathcal{A} s_{+}$is characterized by

$$
\Delta\left(P_{1_{n}}\right)=\sum_{i+j=n} P_{1_{i}} \otimes P_{1_{j}}
$$

Hence, if $\sigma \in S_{n}$, then, in $\bigoplus_{i+j=n} \operatorname{Ind}_{S_{i} \times S_{j}}^{S_{n}} \mathcal{A} s_{+}(i) \otimes \mathcal{A} s_{+}(j)$, we have the identity:

$$
\Delta\left(P_{\sigma}\right)=\Delta\left(\sigma \cdot P_{1_{n}}\right)=\sigma \cdot \Delta\left(P_{1_{n}}\right)=\sum_{i+j=n} \sigma \cdot P_{1_{i}} \otimes P_{1_{j}} .
$$

The unit and the augmentation of $\mathcal{A} s_{+}$are the identity on $\mathcal{A} s_{+}(0)=\mathbf{F}$ (hence, $\mathcal{A} s_{+}$ is a connected Hopf algebra). It should be clear that the product $\mu: \mathcal{A} s_{+} \otimes \mathcal{A} s_{+} \rightarrow$ $\mathcal{A} s_{+}$induces the canonical algebra product on $T(V)$. On the other hand, it is not hard to verify that the map induced by $\Delta$ on $T(V)$ is the shuffle coproduct.

1.2.12. On primitive elements. Let $H$ be a cocommutative Hopf algebra. The primitive part of $H$ is the equalizer

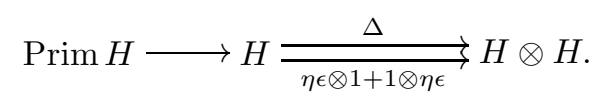

The commutator of $X, Y \in H$ is the element

$$
[X, Y]=\mu(X \otimes Y)-\mu\left(\tau^{*}(X \otimes Y)\right)
$$

where $\tau^{*}$ is the symmetry operator as defined in (1.1.4). We have the following fact, which is well known in the classical theory of Hopf algebras:

1.2.13. Fact. Let $X, Y \in H$. If $X, Y \in \operatorname{Prim} H$, then $[X, Y] \in \operatorname{Prim} H$. 
1.2.14. On commutators in $\mathcal{A} s_{+}$. Let $P_{s} \in \mathcal{A} s_{+}(i)$ and $P_{t} \in \mathcal{A} s_{+}(j)$. It should be clear that the commutator $\left[P_{s}, P_{t}\right]$, as defined above, is the image of the tensor

$$
\left(X_{1} X_{2}-X_{2} X_{1}\right) \otimes P_{s} \otimes P_{t} \in \mathcal{A} s_{+}(2) \otimes \mathcal{A} s_{+}(i) \otimes \mathcal{A} s_{+}(j)
$$

under the operad product

$$
\mathcal{A} s_{+}(2) \otimes \mathcal{A} s_{+}(i) \otimes \mathcal{A} s_{+}(j) \longrightarrow \mathcal{A} s_{+}(i+j) .
$$

In fact, by definition, $\mu\left(P_{s} \otimes P_{t}\right)$ is the image of $X_{1} X_{2} \otimes P_{s} \otimes P_{t}$ under the operad product. Moreover, in $\mathcal{A} s_{+}(2)$, we have $X_{2} X_{1}=\tau \cdot X_{1} X_{2}$. Therefore, by equivariance, the operad product maps $\left(\tau \cdot X_{1} X_{2}\right) \otimes P_{s} \otimes P_{t}$ to $\mu\left(\tau^{*}\left(P_{s} \otimes P_{t}\right)\right)$.

Let $P \in \mathcal{A} s_{+}$belong to the image of the operad embedding $\mathcal{L} i e \hookrightarrow \mathcal{A} s_{+}$. From the observation above, we deduce that $P$ is a sum of iterated commutators of $X_{1} \in \mathcal{A} s_{+}(1)$ by itself. Thus, from the fact 1.2 .13 above, we conclude:

1.2.15. Lemma. The image of the symmetric sequence $\mathcal{L}$ ie under the operad embedding $\mathcal{L}$ ie $\longleftrightarrow \mathcal{A} s_{+}$is contained in $\operatorname{Prim} \mathcal{A} s_{+}$.

Now, the functor $\Gamma: \operatorname{Mod}_{\mathbf{F}}^{S} \longrightarrow \mathcal{F}\left(\operatorname{Mod}_{\mathbf{F}}\right)$ is left exact. Therefore, if $H$ is a Hopf algebra, then we have the identity

$$
\Gamma(\operatorname{Prim} H, V)=\operatorname{Prim} \Gamma(H, V) .
$$

Hence, for $H=\mathcal{A} s_{+}$, we obtain the inclusion

$$
\Gamma(\mathcal{L} i e, V) \subseteq \Gamma\left(\operatorname{Prim} \mathcal{A} s_{+}, V\right)=\operatorname{Prim} T(V) .
$$

Now, the proof of theorem 1.2.5 is complete, because, to recapitulate,

$$
\mathcal{L}_{p}(V) \subseteq \Gamma(\mathcal{L} i e, V) \subseteq \operatorname{Prim} T(V)=\mathcal{L}_{p}(V) .
$$

In addition, since we have $\Gamma(\mathcal{L} i e, V)=\Gamma\left(\operatorname{Prim} \mathcal{A} s_{+}, V\right)$, we can deduce:

1.2.16. Proposition. If the symmetric sequence $\mathcal{A} s_{+}$is equipped with the Hopf algebra structure defined above (cf. 1.2.11), then the operad embedding $\mathcal{L} i e \longleftrightarrow$ $\mathcal{A} s_{+}$identifies $\mathcal{L}$ ie with $\operatorname{Prim} \mathcal{A} s_{+} \subseteq \mathcal{A} s_{+}$.

1.2.17. Poisson algebras. As mentioned earlier, the Poisson operad is the composite of the commutative operad with the Lie operad. In fact, we have a more precise result. The Poisson operad Pois may be built from the commutative operad and the Lie operad by the process of distributive laws (cf. [24]). Explicitly, as a symmetric sequence, $\mathcal{P}$ ois is the composite $\mathcal{C}$ om $\circ \mathcal{L}$ ie. The operad product $\mathcal{P}$ ois $\circ$ Pois $\longrightarrow$ $\mathcal{P}$ ois is the composite of a certain distributive law $\rho: \mathcal{L}$ ie $\circ \mathcal{C}$ om $\longrightarrow \mathcal{C}$ om $\circ$ Lie with the operad product of $\mathcal{C}$ om and $\mathcal{L}$ ie, explicitly:

$$
(\mathcal{C} o m \circ \mathcal{L} i e) \circ(\mathcal{C} o m \circ \mathcal{L} i e) \stackrel{\mathcal{C} o m \circ \rho \circ \mathcal{L} i e}{\longrightarrow}(\mathcal{C o m} \circ \mathcal{C} \text { om }) \circ(\mathcal{L} i e \circ \mathcal{L} i e) \stackrel{\mu \circ \mu}{\longrightarrow} \mathcal{C} \text { om } \circ \mathcal{L} i e .
$$

The distributive law describes how the composite of a Lie operation with a commutative operation may be written as the composite of a commutative operation with a Lie operation. In our case, the commutative operad is generated by the operation $X_{1} X_{2} \in \mathcal{C} \operatorname{om}(2)$ and the Lie operad by the operation $\left[X_{1}, X_{2}\right] \in \mathcal{L} i e(2)$ (cf. [16]). Therefore, it is sufficient to specify the image of $\left[X_{1}, X_{2}\right] \otimes\left(X_{1} X_{2} \otimes X_{1}\right) \in$ $\mathcal{L} i e(2) \otimes(\operatorname{Com}(2) \otimes \mathcal{C}$ om $(1))$ under the distributive law $\rho$. This image is given by the Poisson relation

$$
\left[X_{1} X_{2}, X_{3}\right]=X_{1}\left[X_{2}, X_{3}\right]+\left[X_{1}, X_{3}\right] X_{2} \text {. }
$$

There is a similar process for monads (cf. [1, Section 9.2]). As an example, $T(\rho): T(\mathcal{L} i e) \circ T(\mathcal{C o m}) \longrightarrow T(\mathcal{C o m}) \circ T(\mathcal{L} i e)$ is a distributive law of monads. In 
the same way, $\Gamma(\mathcal{P}$ ois $)$ is the composite $\Gamma(\mathcal{C}$ om $) \circ \Gamma($ Lie $)$ and $\Gamma(\rho)$ is a distributive law, which gives the monad structure of $\Gamma(\mathcal{P}$ ois $)$. Therefore, a $\Gamma \mathcal{P}$ ois-algebra is the "composite" of a $\Gamma \mathcal{C}$ om-algebra with a $\Gamma \mathcal{L} i e$-algebra. The calculation of $\Gamma(\rho)$ determines the distributivity relations between these structures. In characteristic 2, we obtain:

1.2.18. Theorem. Fix a ground field $\mathbf{F}$ of characteristic 2. A (graded) $\Gamma$ Poisalgebra is an $\mathbf{F}$-module equipped with the structure of a (graded) divided power algebra, and with the structure of a (graded) restricted Lie algebra, such that the following distributivity relations are satisfied:

$$
\begin{gathered}
\text { (1) }[x y, z]=x[y, z]+[x, z] y, \quad(2) \quad\left[\gamma_{n}(x), y\right]=\gamma_{n-1}(x)[x, y], \\
(3) \quad F(x y)=x y[x, y] \quad \text { and } \quad(4) \quad F\left(\gamma_{2}(x)\right)=0 .
\end{gathered}
$$

(The letter $F$ denotes the Frobenius operation of the restricted Lie algebra structure.)

Proof. For simplicity, we work with ungraded objects. The Lie bracket and the Frobenius generate the Lie operations. The commutative product and the second divided power generate the commutative operations. Therefore, it is sufficient to write down the distributivity relations for these operations.

Relation (1) is clear. The problem is to show relations (2), (3), and (4). We calculate the image under the operad product

$$
\Gamma(\mathcal{P} \text { ois }, \Gamma(\mathcal{P} \text { ois }, V)) \longrightarrow \Gamma(\mathcal{P} \text { ois }, V)
$$

of the left-hand side and of the right-hand side of each equation. We check that these images agree.

Proof of relation (2). It is sufficient to prove the relation in $\Gamma(\mathcal{P}$ ois,$V)$, for $V=$ $\operatorname{Span}\langle x, y\rangle$.

In $\Gamma(\mathcal{P}$ ois,$V)$, we have $\gamma_{n}(x)=X_{1} \cdots X_{n} \otimes x^{\otimes n}$. By (1.1.20), we obtain

$$
\left[\gamma_{n}(x), y\right]=\sum_{s \in S_{n+1} / S_{n} \times S_{1}} s \cdot\left[X_{1} \cdots X_{n}, X_{n+1}\right] \otimes s_{*}\left(x^{\otimes n} \otimes y\right) .
$$

In this sum, the coefficient of the word $x^{\otimes n} \otimes y$, is the polynomial

$$
\left[X_{1} \cdots X_{n}, X_{n+1}\right]=\sum_{i} X_{1} \cdots \widehat{X}_{i} \cdots X_{n}\left[X_{i}, X_{n+1}\right]
$$

By $S_{n+1}$-invariance, the other terms of the sum can be deduced from this one.

On the other hand, we have $[x, y]=\left[X_{1}, X_{2}\right] \otimes(x \otimes y)+\left[X_{1}, X_{2}\right] \otimes(y \otimes x)$, and hence, by (1.1.20),

$$
\begin{aligned}
\gamma_{n-1}(x)[x, y]= & \sum_{s \in S_{n+1} / S_{n-1} \times S_{2}} s \cdot X_{1} \cdots X_{n-1}\left[X_{n}, X_{n+1}\right] \\
& \otimes s_{*}\left(x^{\otimes n-1} \otimes x \otimes y+x^{\otimes n-1} \otimes y \otimes x\right) .
\end{aligned}
$$

The $(n-1,2)$-shuffles form a set of representatives of $S_{n+1} / S_{n-1} \times S_{2}$. An $(n-1,2)$ shuffles such that $s_{*}\left(x^{\otimes n-1} \otimes x \otimes y\right)=x^{\otimes n} \otimes y$ is specified by $s(n)=i<n+1$, $s(n+1)=n+1$. There is no $(n-1,2)$-shuffle such that $s_{*}\left(x^{\otimes n-1} \otimes y \otimes x\right)=$ $x^{\otimes n-1} \otimes x \otimes y$, because this identity would imply $s(n)=n+1$. Therefore, the coefficient of $x^{\otimes n} \otimes y$ in the expansion of $\gamma_{n-1}(x)[x, y]$ is the sum

$$
\sum_{s} s \cdot X_{1} \cdots X_{n-1}\left[X_{n}, X_{n+1}\right]
$$


where $s$ ranges over the set of $(n-1,2)$-shuffles, which fix $n+1$.

Finally, the coefficients of $x^{\otimes n} \otimes y$ in $\left[\gamma_{n}(x), y\right]$ and $\gamma_{n-1}(x)[x, y]$ are equal and identity (2) follows by $S_{n+1}$-invariance.

Proof of relation (3). Once again, it is sufficient to prove the relation in $\Gamma(\mathcal{P}$ ois, $V)$, for $V=\operatorname{Span}\langle x, y\rangle$.

Let us calculate $F(x y)$ in $\Gamma\left(\mathcal{P}_{\text {ois }}, V\right)$. In $\Gamma\left(\mathcal{P}_{\text {ois }}, V\right)$, the product $x y$ is given by the formula $x y=X_{1} X_{2} \otimes(x \otimes y+y \otimes x)$ and $F(x y)$ is the image of $\left[X_{1}, X_{2}\right] \otimes(x y)^{\otimes 2}$ under the monad product

$$
\Gamma\left(\mathcal{P} \text { ois }, \Gamma\left(\mathcal{P}_{\text {ois }}, V\right)\right) \longrightarrow \Gamma(\mathcal{P} \text { ois }, V) .
$$

We go back to the definition of this monad product (cf. 1.1.18). We obtain

$$
F(x y)=\sum_{s \in S_{4} / S_{2}} s \cdot\left[X_{1} X_{2}, X_{3} X_{4}\right] \otimes s_{*}(x \otimes y \otimes x \otimes y) .
$$

(We consider the embedding $S_{2} \longleftrightarrow S_{4}$, which maps $s$ to the block permutation $s(2,2)$.) Let $G$ be the subgroup of permutations, which fix the tensor $x \otimes y \otimes x \otimes$ $y$. The polynomial $P=\sum_{s \in G / S_{2}} s \cdot\left[X_{1} X_{2}, X_{3} X_{4}\right]$ is the coefficient of the word $x \otimes y \otimes x \otimes y$ in the expansion of $F(x y)$. We have $G=\left\{1,\left(\begin{array}{ll}13 \\ 3\end{array}\right),(24),(13)(24)\right\}$. Moreover, $\left(\begin{array}{ll}13 \\ 3\end{array}\right)(24)$ is the block transposition $\tau(2,2)$, and hence, $1,(13)$ is a set of representatives of $G / S_{2}$. Thus, we obtain

$$
\begin{aligned}
P & =\left[X_{1} X_{2}, X_{3} X_{4}\right]+\left[X_{2} X_{3}, X_{1} X_{4}\right] \\
& =X_{1} X_{2}\left[X_{3}, X_{4}\right]+X_{1} X_{4}\left[X_{2}, X_{3}\right]+X_{2} X_{3}\left[X_{1}, X_{4}\right]+X_{3} X_{4}\left[X_{1}, X_{2}\right] .
\end{aligned}
$$

On the other hand, by (1.1.20), $x y[x, y]$, which is the product of

$$
X_{1} \otimes x, X_{1} \otimes y,\left[X_{1}, X_{2}\right] \otimes(x \otimes y+y \otimes x) \in \Gamma(\mathcal{P} \text { ois }, V),
$$

is given by the formula

$$
\sum_{s \in S_{4} / S_{1} \times S_{1} \times S_{2}} s \cdot X_{1} X_{2}\left[X_{3}, X_{4}\right] \otimes s_{*}(x \otimes y \otimes x \otimes y+x \otimes y \otimes y \otimes x) .
$$

As representatives of $S_{4} / S_{1} \times S_{1} \times S_{2}$, we take the $(1,1,2)$-shuffles. The permutations $s=1,\left(\begin{array}{ll}1 & 3\end{array}\right),\left(\begin{array}{l}1 \\ 1\end{array}\right)\left(\begin{array}{l}2 \\ 4\end{array}\right)$ are the $(1,1,2)$-shuffles, which fix $x \otimes y \otimes x \otimes y$, and $s=\left(\begin{array}{lll}2 & 4 & 3\end{array}\right)$ is the unique $(1,1,2)$-shuffle such that $s_{*}(x \otimes y \otimes y \otimes x)=x \otimes y \otimes x \otimes y$. Therefore, the coefficient of $x \otimes y \otimes x \otimes y$ in the expansion of $x y[x, y]$ is the polynomial

$$
\begin{aligned}
& \left(1+(13)+\left(\begin{array}{ll}
1 & 3
\end{array}\right)(24)+(243)\right) \cdot X_{1} X_{2}\left[X_{3}, X_{4}\right] \\
& \quad=X_{1} X_{2}\left[X_{3}, X_{4}\right]+X_{3} X_{2}\left[X_{1}, X_{4}\right]+X_{3} X_{4}\left[X_{1}, X_{2}\right]+X_{1} X_{4}\left[X_{2}, X_{3}\right] .
\end{aligned}
$$

As in the proof of relation (2), by $S_{4}$-invariance, it follows that $F(x y)=x y[x, y]$.

Proof of relation (4). We prove the relation in $\Gamma(\mathcal{P}, V)$, where $V=\operatorname{Span}\langle x\rangle$. As in the proof of relation (3), going back to the definition of the monad product, we obtain

$$
F\left(\gamma_{2}(x)\right)=\sum_{s} s \cdot\left[X_{1} X_{2}, X_{3} X_{4}\right] \otimes x^{\otimes 4},
$$

where $s$ ranges over the set of $(2,2)$-shuffles such that $s(1)<s(3)$. Thus, $s=1$, (2 3), or (2 43 ). We have

$$
\begin{aligned}
{\left[X_{1} X_{2}, X_{3} X_{4}\right] } & =X_{1} X_{3}\left[X_{2}, X_{4}\right]+X_{1} X_{4}\left[X_{2}, X_{3}\right] \\
& +X_{2} X_{3}\left[X_{1}, X_{4}\right]+X_{2} X_{4}\left[X_{1}, X_{3}\right]
\end{aligned}
$$


and by an immediate calculation, one may check the identity

$$
\left(1+\left(\begin{array}{ll}
2 & 3
\end{array}\right)+\left(\begin{array}{lll}
2 & 4 & 3
\end{array}\right)\right) \cdot\left[X_{1} X_{2}, X_{3} X_{4}\right]=0 .
$$

1.2.19. On $\Gamma$ Pois-algebras in odd characteristic. In odd characteristic, we have again

$$
\begin{gathered}
\text { (1) }[x y, z]=x[y, z]+[x, z] y, \quad(2) \quad\left[\gamma_{n}(x), y\right]=\gamma_{n-1}(x)[x, y] \\
\text { and } \quad(4) \quad F\left(\gamma_{p}(x)\right)=0 .
\end{gathered}
$$

But it is more difficult to write down relation (3) explicitly. As in 6], one may show that we have a distributivity relation of the form

$$
\text { (3) } F(x y)=\sum_{0 \leq i, j, i+j \leq p} x^{i} y^{j} \Gamma_{i, j},
$$

where $\Gamma_{i, j}$ are products of Lie polynomials in $x$ and $y$. Furthermore, these polynomials verify the relations

$$
(i-j)[x, y] \Gamma_{i, j}=\left[\Gamma_{i, j-1}, x\right]+\left[\Gamma_{i-1, j}, y\right]
$$

(see [6, pp. 336-337]).

\section{HOMOTOPY OPERATIONS}

2.1. General theory and classical results. We recall some classical results on the nonlinear derived functors of Dold and Puppe. In particular, we show that the derived functor of a monad is equipped with a canonical monad structure. This gives us the right framework for the construction of homotopy operations from algebraic structures.

Let $V$ be a simplicial module. We denote the associated chain complex by $C(V)$ and the normalized chain complex by $N(V)$. By definition

$$
N_{n}(V):=\bigcap_{0<i \leq n} \operatorname{ker}\left(d_{i}: V_{n} \longrightarrow V_{n-1}\right) .
$$

We denote by $\pi_{*}(V)$ the homotopy of $V$, which is defined as the homology of the chain complex $C(V)$, or equivalently as the homology of the chain complex $N(V)$.

Recall that we have a canonical decomposition

$$
V_{n}=N_{n}(V) \oplus\left(\sum_{i=0}^{n-1} s_{i} V_{n-1}\right) .
$$

Moreover, $N$ provides an equivalence between the category of simplicial Fmodules and the category of chain complexes. The inverse equivalence is denoted by $K$ (see [10] or [25] Chapter V]).

2.1.1. The "derived functor" of a nonadditive functor. Any functor $T: \operatorname{Mod}_{\mathbf{F}} \rightarrow$ $\operatorname{Mod}_{\mathbf{F}}$ extends to a functor $T: s \operatorname{Mod}_{\mathbf{F}} \rightarrow s \operatorname{Mod}_{\mathbf{F}}$. We denote by $T_{*}: n \operatorname{Mod}_{\mathbf{F}} \rightarrow$ $n \operatorname{Mod}_{\mathbf{F}}$ the functor defined by $T_{*}(V):=\pi_{*} T(K V)$. (Recall that $s \operatorname{Mod}_{\mathbf{F}}$ denotes the category of simplicial modules and $n \operatorname{Mod}_{\mathbf{F}}$ the category of graded modules.) The functor $T_{*}$, also known as the derived functor of $T$, was introduced by Dold and Puppe in [10] (see also [2], [3]). 
2.1.2. Theorem (See [8 Remark 4.5]). Let $T: \operatorname{Mod}_{\mathbf{F}} \longrightarrow \operatorname{Mod}_{\mathbf{F}}$ be a functor. There is an isomorphism

$$
\phi_{T, X}: T_{*}\left(\pi_{*}(X)\right) \stackrel{\simeq}{\longrightarrow} \pi_{*}(T(X)),
$$

which is natural in $T \in \mathcal{F}\left(\operatorname{Mod}_{\mathbf{F}}\right)$ and in $X \in s \operatorname{Mod}_{\mathbf{F}}$.

2.1.3. The natural isomorphism $\phi_{T, X}$. We specify such a natural isomorphism $\phi_{T, X}$. Let $V$ be a graded module. We have a canonical isomorphism $V \stackrel{\simeq}{\longrightarrow} \pi_{*}(K V)$. We set $\phi_{T, K V}$ to be the composite

$$
T_{*}\left(\pi_{*}(K V)\right) \stackrel{\simeq}{\longleftarrow} T_{*}(V)=\pi_{*}(T(K V)) .
$$

This property uniquely determines the natural isomorphism $\phi_{T, X}$. More specifically, we shall use the following observation (see fact 2.1.6 below). Let $X$ be a simplicial module. Let $C$ be a graded module equipped with a map $c^{\prime}: C \longrightarrow \pi_{*}(X)$. Fix a morphism of chain complexes $c: C \longrightarrow N(T(K V))$ which induces $c^{\prime}$ in homotopy. Then, $c$ is equivalent to a morphism of simplicial modules $c^{\prime \prime}: K V \longrightarrow X$. Now, $\phi_{T, X}$ is supposed to be natural in $X$. It follows that the diagram

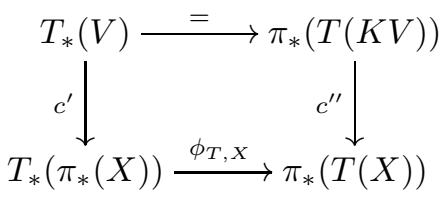

commutes.

2.1.4. Proposition. Let $S, T: \operatorname{Mod}_{\mathbf{F}} \longrightarrow \operatorname{Mod}_{\mathbf{F}}$. There is a unique functor isomorphism

$$
a_{S, T}: S_{*} T_{*} \stackrel{\simeq}{\longrightarrow}(S T)_{*},
$$

which makes the following diagram commute:

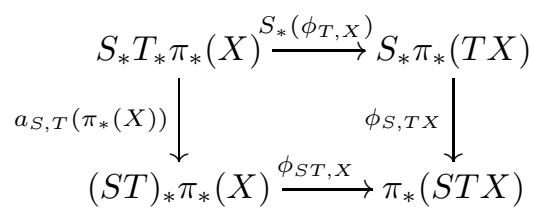

for each simplicial module $X$. Moreover, $a_{S, T}$ is unital and associative; we have $a_{I, T}=a_{T, I}=1$ and $a_{S, T U} \cdot S_{*} a_{S, T}=a_{S T, U} \cdot a_{S, T} U_{*}$.

2.1.5. The natural isomorphism $a_{S, T}$. In fact, if $V$ is a graded module, then the $\operatorname{map} a_{S, T}(V): S_{*} T_{*}(V) \longrightarrow(S T)_{*}(V)$ is necessarily

$$
S_{*}\left(T_{*}(V)\right)=S_{*}\left(\pi_{*}(T(K V))\right) \stackrel{\phi_{S, T(K V)}}{\longrightarrow} \pi_{*}(S(T(K V)))=(S T)_{*}(V) .
$$

Thus, if we fix the morphism $\phi$ as in (2.1.3), then we obtain

2.1.6. Fact. Let $C$ be a graded module equipped with a map $c^{\prime}: V \longrightarrow T_{*}(V)$. Fix a morphism of chain complexes $c: C \longrightarrow N(T(K V))$ which induces $c^{\prime}$ in homotopy. Then, $c$ is equivalent to a simplicial morphism $c^{\prime \prime}: K C \longrightarrow T(K V)$. 
We claim that the diagram

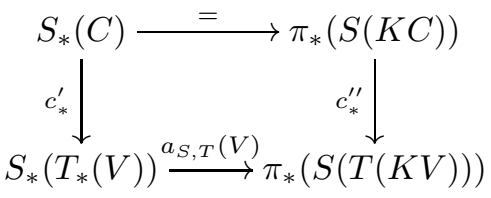

commutes.

2.1.7. Homotopy operations arising from algebraic structures. If $T$ is a monad, then the previous proposition implies that $T_{*}$ is also a monad. If $A$ is a simplicial $T$ algebra, then $\pi_{*}(A)$ is a $T_{*}$-algebra. There is an explicit description of the structure of a $T_{*}$-algebra in the following cases:

- $T$ is the free commutative algebra functor (see [11, [18] and [17, Section II.2] for the case Char $\mathbf{F}=2$, and [3], for the general case);

- $T$ is the free restricted Lie algebra functor (see [4] and [7]).

Assume $C h a r \mathbf{F}=2$. If $A$ is a simplicial commutative algebra, then there are nontrivial operations

$$
\delta_{i}: \pi_{n}(A) \longrightarrow \pi_{n+i}(A) \text { for } 2 \leq i \leq n .
$$

Moreover, as mentioned in the introduction, $\pi_{*}(A)$ is equipped with a structure of a divided power algebra. In fact, the $\delta$-operations are also known as higher divided squares, because the top $\delta$-operation $\delta_{n}: \pi_{n}(A) \longrightarrow \pi_{2 n}(A)$ is the divided square $\gamma_{2}$ of $\pi_{*}(A)$. Similarly, if $g$ is a 2-restricted simplicial Lie algebra, then there are non-trivial operations

$$
\lambda_{i}: \pi_{n}(g) \longrightarrow \pi_{n+i}(g) \text { for } 0 \leq i \leq n .
$$

Moreover, $\pi_{*}(g)$ is equipped with a structure of a 2-restricted Lie algebra. In fact, the top $\lambda$-operation $\lambda_{n}: \pi_{n}(g) \longrightarrow \pi_{2 n}(g)$ is the Frobenius of $\pi_{*}(g)$.

By the Künneth formula, we have $\pi_{*} T(\mathcal{A} s, V)=T\left(\mathcal{A} s, \pi_{*}(V)\right)$. Hence, we have $T(\mathcal{A} s)_{*}=T(\mathcal{A} s)$. (Recall that $T(\mathcal{A} s)$ extends to a functor on the category of graded $\mathbf{F}$-modules.) More generally, if $\mathcal{P}$ is projective as a symmetric sequence, then there are no nontrivial homotopy operations for simplicial $\mathcal{P}$-algebras.

Similarly, the structure of a coalgebra gives rise to homotopy operations known as Steenrod operations (see [9]). If $H$ is a simplicial Hopf algebra, then $\pi_{*}(H)$ is equipped with both operations arising from its algebra structure and Steenrod operations arising from its coalgebra structure. The distributivity relations between these operations have been calculated (cf. [29]).

To conclude, let us mention that many spectral sequences in algebraic topology are equipped with a compatible action of operations of the nature above (see [12], [30]).

2.2. Homotopy operations for simplicial algebras over an operad. We study the structure of the derived functor of $T(M,-)$, where $M$ denotes a symmetric sequence. The main point is to understand the symmetries inherent to the shuffle map.

2.2.1. The shuffle map. Let $V_{1}, \ldots, V_{r}$ be simplicial modules. The shuffle map is a natural chain equivalence

$$
\nabla: C\left(V_{1}\right) \otimes \cdots \otimes C\left(V_{r}\right) \longrightarrow C\left(V_{1} \otimes \cdots \otimes V_{r}\right)
$$


which is strictly associative and commutative (cf. [22, Chapter 8] or [10]). Let us state precisely the facts which are used in this paper.

Let $I=(I(1), \ldots, I(r))$ be an ordered partition of some ordinal $\{0, \ldots, n-1\}$. Below, we associate to $I$ a natural transformation

$$
\nabla_{I}: C\left(V_{1}\right) \otimes \cdots \otimes C\left(V_{r}\right) \longrightarrow C\left(V_{1} \otimes \ldots \otimes V_{r}\right) .
$$

Recall that an ordered partition $I=(I(1), \ldots, I(r))$ which has $\# I(1)=\alpha(1), \ldots$, $\# I(r)=\alpha(r)$ is equivalent to an $(\alpha(1), \ldots, \alpha(r))$-shuffle. The sign of the partition $I$, denoted by $\operatorname{sgn}(I)$, is the signature of the associated shuffle. The shuffle map is defined as the sum

$$
\nabla:=\sum_{I} \operatorname{sgn}(I) \cdot \nabla_{I}
$$

where $I$ ranges over the set of all ordered partitions.

Let $I$ be as above. In fact, we define a map

$$
\nabla_{I}: C_{\alpha(1)}\left(V_{1}\right) \otimes \cdots \otimes C_{\alpha(r)}\left(V_{r}\right) \longrightarrow C_{n}\left(V_{1} \otimes \cdots \otimes V_{r}\right)
$$

and we set the map $\nabla_{I}$ to be zero on the other components. For $p=1, \ldots, r$, the complement of $I(p)$ in $\{0, \ldots, n-1\}$ is denoted by $I^{\prime}(p)$. Let $C=\left\{c_{1}<\cdots<c_{t}\right\}$ be any $I^{\prime}(1), \ldots, I^{\prime}(r)$. We denote by $\sigma_{C}$ the composite $\sigma_{c_{1}} \cdots \sigma_{c_{t}}$ in the simplicial category. We define $\nabla_{I}$ as the tensor product $\sigma_{I^{\prime}(1)}^{*} \otimes \cdots \otimes \sigma_{I^{\prime}(r)}^{*}$.

2.2.2. The operad of ordered partitions. In the sequel, we denote by $\Pi(r)$ the set of ordered partitions of all finite ordinals into $r$ components. In the next paragraphs, we show that $\Pi$ is equipped with a kind of operad structure.

2.2.3. Composition of partitions and associativity of the shuffle map. We have mentioned that the shuffle map is associative. Nevertheless, in order to prove certain associativity relations (see lemma 2.2.15), we need a stronger result, which we express as follows.

There is a (partially defined) composition product

$$
\Pi(r) \times \Pi\left(s_{1}\right) \times \cdots \times \Pi\left(s_{r}\right)--\rightarrow \Pi\left(s_{1}+\cdots+s_{r}\right) .
$$

The composite $K=I\left(J_{1}, \ldots, J_{r}\right)$ is defined as follows. We assume that $I=$ $(I(1), \ldots, I(r))$ is an ordered partition of $\{0, \ldots, n-1\}$ such that $\# I(p)=\alpha(p)$. We assume that $J_{p}=\left(J_{p}(1), \ldots, J_{p}\left(s_{p}\right)\right)$ is an ordered partition of $\{0, \ldots, \alpha(p)-1\}$ such that $\# J_{p}(q)=\beta_{p}(q)$. Then

$$
K=\left(K(1,1), \ldots, K\left(1, s_{1}\right), \ldots, K(r, 1), \ldots, K\left(r, s_{r}\right)\right)
$$

is an ordered partition of $\{0, \ldots, n-1\}$ such that $\# K(p, q)=\beta_{p}(q)$. If $I(p)=$ $\{i(1)<\cdots<i(\alpha)\}$ and $J_{p}(q)=\{j(1)<\cdots<j(\beta)\}$, then we define $K(p, q):=$ $\{i(j(1))<\cdots<i(j(\beta))\}$. In fact, if $s$ (resp. $\left.t_{1}, \ldots, t_{r}\right)$ is the shuffle associated to the partition $I$ (resp. $\left.J_{1}, \ldots, J_{r}\right)$. then $s \cdot t_{1} \oplus \cdots \oplus t_{r}$ is a shuffle and $I\left(J_{1}, \ldots, J_{r}\right)$ is the partition associated to this shuffle.

Now, the associativity of the shuffle map follows from the following fact:

2.2.4. Fact. Whenever it makes sense, we have the identity

$$
\nabla_{I} \cdot\left(\nabla_{J_{1}} \otimes \cdots \otimes \nabla_{J_{r}}\right)=\nabla_{I\left(J_{1}, \ldots, J_{r}\right)} .
$$


2.2.5. Invariance under the action of the symmetric group. The set of ordered partition $\Pi(r)$ is equipped with the obvious action of the symmetric group $S_{r}$ : if $I=(I(1), \ldots, I(r)) \in \Pi(r)$ and $\sigma \in S_{r}$, then $\sigma^{*} I$ is the partition $\sigma^{*} I=$ $(I(\sigma(1)), \ldots, I(\sigma(r)))$. In fact, if $s$ is the $(\alpha(1), \ldots, \alpha(r))$-shuffle associated to $I$, then the composite

$$
s \cdot \sigma^{-1}(\alpha(1), \ldots, \alpha(r))
$$

is an $(\alpha(\sigma(1)), \ldots, \alpha(\sigma(r)))$-shuffle and $\sigma^{*} I$ is the partition associated to this shuffle. Recall that

$$
\sigma^{*}: C\left(V_{1}\right) \otimes \cdots \otimes C\left(V_{r}\right) \rightarrow C\left(V_{\sigma(1)}\right) \otimes \cdots \otimes C\left(V_{\sigma(r)}\right),
$$

and

$$
\sigma^{*}: C\left(V_{1} \otimes \cdots \otimes V_{r}\right) \rightarrow C\left(V_{\sigma(1)} \otimes \cdots \otimes V_{\sigma(r)}\right)
$$

denote the natural transformations given by place permutation.

We have mentioned that the shuffle map is commutative. This property follows from the following fact:

2.2.6. Fact. Let $I$ be a partition of $\{0, \ldots, n-1\}$ into $r$ components. Let $\sigma \in S_{r}$. We have the identity

$$
\sigma^{*} \cdot \operatorname{sgn} I \cdot \nabla_{I}=\operatorname{sgn}\left(\sigma^{*} I\right) \cdot \nabla_{\sigma^{*} I} \cdot \sigma^{*} .
$$

2.2.7. The homotopy morphisms induced by the shuffle map. Fix a symmetric sequence $M$ and a graded module $V$. Since the shuffle map is equivariant with respect to the action of the symmetric group, it induces a simplicial morphism

$$
\nabla: K T(M, V) \longrightarrow T(M, K V) .
$$

Hence, in homotopy, we obtain

$$
\nabla_{*}: T(M, V) \longrightarrow \pi_{*} T(M, K V)=T(M)_{*}(V) .
$$

Similarly, the shuffle map induces a simplicial morphism

$$
\nabla: K \Gamma(M, V) \longrightarrow \Gamma(M, K V)
$$

which gives in homotopy

$$
\nabla_{*}: \Gamma(M, V) \longrightarrow \pi_{*} \Gamma(M, K V)=\Gamma(M)_{*}(V) .
$$

The next lemma follows from the associativity of the shuffle map. We do not give the proof of this lemma which is similar to the proof of lemma 2.2 .15 below.

2.2.8. Lemma. Let $F(M,-)$ denote either the functor $T(M,-)$ or the functor $\Gamma(M,-)$. The natural transformation defined above $\nabla_{*}: F(M, V) \longrightarrow F(M)_{*}(V)$ is compatible with the composition of symmetric sequences. Explicitly, the diagram

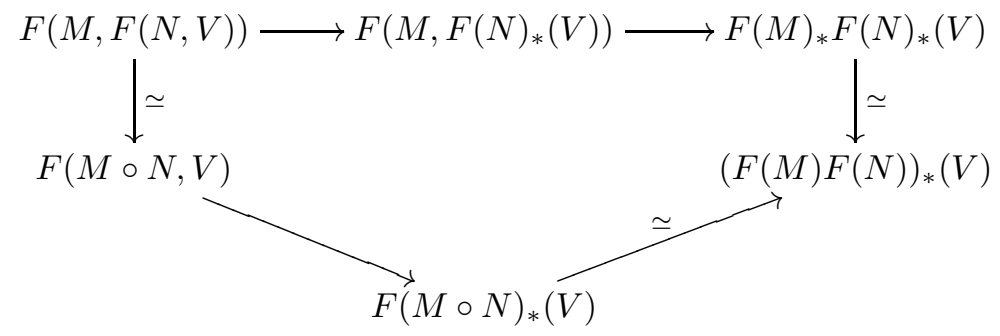

is commutative. 
As a consequence, we obtain

2.2.9. Proposition. Let $\mathcal{P}$ be a connected operad.

1) The natural transformation $\nabla_{*}: T(\mathcal{P}, V) \longrightarrow T(\mathcal{P})_{*}(V)$ (respectively, $\nabla_{*}$ : $\left.\Gamma(\mathcal{P}, V) \longrightarrow \Gamma(\mathcal{P})_{*}(V)\right)$ is a morphism of monads.

2) If $A$ is a simplicial $\mathcal{P}$-algebra (respectively, $\Gamma \mathcal{P}$-algebra), then $\pi_{*} A$ is equipped with the structure of a graded $\mathcal{P}$-algebra (resp. $\Gamma \mathcal{P}$-algebra).

In fact, if $A$ is a simplicial $\mathcal{P}$-algebra, then the $\mathcal{P}$-algebra structure of $\pi_{*}(A)$ should be clear. The $\mathcal{P}$-algebra product of $\pi_{*}(A)$ is induced by the composite of the $\mathcal{P}$-algebra product of $A$ with the shuffle map

$$
\mathcal{P}(r) \otimes C(A)^{\otimes r} \stackrel{\nabla}{\longrightarrow} \mathcal{P}(r) \otimes C\left(A^{\otimes r}\right) \longrightarrow C(A) .
$$

The product $\mathcal{P}(r) \otimes \pi_{*}(A)^{\otimes r} \longrightarrow \pi_{*}(A)$ is associative and $S_{r}$-invariant because the shuffle map is.

If $A$ is a $\Gamma \mathcal{P}$-algebra, then it is less obvious that $\pi_{*}(A)$ is a $\Gamma \mathcal{P}$-algebra, because there is no direct map from $\left(\mathcal{P}(r) \otimes \pi_{*}(A)^{\otimes r}\right)^{S_{r}}$ to $\pi_{*}\left(\mathcal{P}(r) \otimes A^{\otimes r}\right)^{S_{r}}$.

A graded module $V$ is 2-reduced if $V_{0}=V_{1}=0$. If $V$ is 2-reduced, then it is not hard to see that $T(\mathcal{P})_{*}(V)$ is 2 -reduced. Therefore, $T(\mathcal{P})_{*}$ restricts to a monad on the category of the 2-reduced graded modules. If $V$ is a simplicial module, then $\pi_{* \geq 2}(V)$ denotes the graded module $\bigoplus_{i \geq 2} \pi_{i}(V)$.

The following theorem is the main result of this paper.

2.2.10. Theorem. Let $\mathcal{P}$ be a connected operad. For $V$ 2-reduced, there is a morphism of monads

$$
\nabla_{*}^{0}: \Gamma(\mathcal{P}, V) \longrightarrow T(\mathcal{P})_{*}(V)
$$

which fits into the commutative diagram

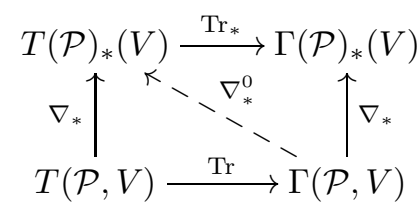

The proof of this theorem is postponed to the end of this section. Recall that $\operatorname{Tr}: T(\mathcal{P}, V) \longrightarrow \Gamma(\mathcal{P}, V)$ is the morphism of monads given by the norm map. In homotopy, the norm map induces a morphism of monads $\operatorname{Tr}_{*}: T(\mathcal{P})_{*}(V) \longrightarrow$ $\Gamma(\mathcal{P})_{*}(V)$. It is easy to see that the solid part (the frame) of the diagram of the theorem commutes.

2.2.11. Corollary. If $A$ is a simplicial $\mathcal{P}$-algebra, then the $\mathcal{P}$-algebra structure of $\pi_{* \geq 2}(A)$ extends to a structure of a graded $\Gamma \mathcal{P}$-algebra.

As an immediate consequence, by theorem 1.2.5 and theorem 1.2.3, we obtain

2.2.12. Corollary. 1) If $A$ is a simplicial commutative algebra, then $\pi_{* 22}(A)$ is equipped with a structure of a graded divided power algebra.

2) If $g$ is a connected simplicial Lie algebra, then $\pi_{* \geq 2}(g)$ is equipped with a structure of a graded restricted Lie algebra.

In the case of commutative algebras, we recover the divided power operations introduced by Cartan in [5]. 
It follows from theorem 2.2.10 that the morphism $\nabla_{*}: T(\mathcal{P}, V) \longrightarrow T(\mathcal{P})_{*}(V)$ is not an embedding in general; the next proposition implies that the morphism $\nabla_{*}^{0}: \Gamma(\mathcal{P}, V) \longrightarrow T(\mathcal{P})_{*}(V)$ is.

2.2.13. Proposition. Let $M$ be a symmetric sequence. The morphism

$$
\nabla_{*}: \Gamma(M, V) \longrightarrow \Gamma(M)_{*}(V)
$$

is an embedding.

Proof. Fix $\gamma \in \Gamma(M, V)$. In regard to the proposition, we may assume that $\gamma$ is homogeneous. Explicitly, $\gamma \in\left(M(n) \otimes V^{\otimes n}\right)^{S_{n}}$, where $n \in \mathbf{N}$. Furthermore, we may assume that $\gamma$ has an expansion of the form

$$
\gamma=\sum_{\sigma \in S_{n} / S_{\alpha}}(\sigma x) \otimes \sigma_{*}\left(v_{1}^{\otimes \alpha_{1}} \cdots v_{r}^{\otimes \alpha_{r}}\right)
$$

where $v_{1}, \ldots, v_{r}$ are linearly independent elements of $V$ and $x \in M(n)^{S_{\alpha}}$. In this way, the $S_{n}$-module generated by $\left(v_{1}^{\otimes \alpha_{1}} \cdots v_{r}^{\otimes \alpha_{r}}\right)$ is a direct summand of $V^{\otimes n}$.

By definition, $\nabla_{*}(\gamma)$ is represented by the cycle

$$
\left.\nabla(\gamma)=\sum \operatorname{sgn}(I)(\sigma x) \otimes \nabla_{I} \sigma_{*}\left(v_{1}^{\otimes \alpha_{1}} \cdots v_{r}^{\otimes \alpha_{r}}\right)\right) \in N\left(\left(M(n) \otimes(K V)^{\otimes n}\right)^{S_{n}}\right)
$$

(see 2.2.7). Let $K v_{i} \subseteq K V$ denote the image of the $\mathbf{F}$-module generated by $v_{i}$ under the $K$ functor. Clearly, $\nabla(\gamma)$ is an invariant tensor in the $S_{n}$-module generated by

$$
N\left(M(n) \otimes\left(K v_{1}\right)^{\otimes \alpha_{1}} \otimes \cdots \otimes\left(K v_{r}\right)^{\otimes \alpha_{r}}\right) \subseteq N\left(M(n) \otimes(K V)^{\otimes n}\right) .
$$

Furthermore, $\gamma$ has degree $\alpha_{1}\left|v_{1}\right|+\cdots+\alpha_{r}\left|v_{r}\right|$. Now, observe that the chain complex $N_{t}\left(\left(K v_{1}\right)^{\otimes \alpha_{1}} \otimes \cdots \otimes\left(K v_{r}\right)^{\otimes \alpha_{r}}\right)$ vanishes in degree $t>\alpha_{1}\left|v_{1}\right|+\cdots+\alpha_{r}\left|v_{r}\right|$. Hence, if $\nabla_{*}(\gamma)$ vanishes in $\pi_{*}(\Gamma(M, K V))$, then $\nabla(\gamma)$ is null in $N(\Gamma(M, K V))$. To conclude, observe that

$$
\left.\nabla(\gamma)=\sum \operatorname{sgn}(I)(\sigma x) \otimes \nabla_{I} \sigma_{*}\left(v_{1}^{\otimes \alpha_{1}} \cdots v_{r}^{\otimes \alpha_{r}}\right)\right)=0
$$

implies

$$
x \otimes\left(v_{1}^{\otimes \alpha_{1}} \cdots v_{r}^{\otimes \alpha_{r}}\right)=0 .
$$

(Recall that $v_{1}, \ldots, v_{n}$ are supposed to be linearly independent.)

Theorem 2.2.10 is an immediate consequence of the next lemmas. More precisely, lemma 2.2.14 shows the existence of the map $\nabla_{*}^{0}: \Gamma(\mathcal{P}, V) \longrightarrow T(\mathcal{P})_{*}(V)$ and lemma 2.2.15 implies that $\nabla_{*}^{0}$ is a monad morphism.

2.2.14. Lemma. Let $V$ be a 2-reduced graded module. There is a unique simplicial morphism $\nabla_{0}: K \Gamma(M, V) \longrightarrow T(M, K V)$ which makes the following diagram commute:

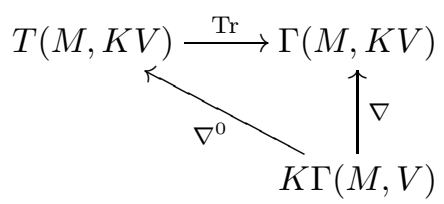

Proof. This lemma follows from the following observation: the symmetric group $S_{r}$ acts freely on the set of partitions $I=(I(1), \ldots, I(r))$ such that

$$
I(k) \neq \emptyset, \forall k=1, \ldots, r \text {. }
$$


Let $\gamma \in \Gamma(M, V)$. We may assume $\gamma=\sum x^{i} \otimes\left(v_{(1)}^{i} \otimes \cdots \otimes v_{(r)}^{i}\right)$ with $x^{i} \in M(r)$ and $v_{(k)}^{i} \in V$. Recall that $\nabla(\gamma) \in \Gamma(M, V)$ is given by the formula

$$
\nabla(\gamma)=\sum_{I \in \Pi(r)} \operatorname{sgn}(I) \cdot x^{i} \otimes \nabla_{I}\left(v_{(1)}^{i} \otimes \cdots \otimes v_{(r)}^{i}\right) .
$$

By hypothesis, we have $\left|v_{(k)}^{i}\right| \geq 1$. Therefore, the observation above implies that $\nabla(\gamma)$ belongs to a submodule of $M(r) \otimes(K V)^{\otimes r}$ on which the symmetric group acts freely. As a consequence, there is a unique $\nabla^{0}(\gamma) \in T(M, K V)$ such that $\operatorname{Tr} \nabla^{0}(\gamma)=\nabla(\gamma)$. Explicitly, we have

$$
\nabla^{0}(\gamma)=\sum_{I \in \Pi(r) / S_{r}} \operatorname{sgn}(I) \cdot x^{i} \otimes \nabla_{I}\left(v_{(1)}^{i} \otimes \cdots \otimes v_{(r)}^{i}\right)
$$

( $I$ ranges over any set of representatives of the quotient $\left.\Pi(r) / S_{r}\right)$.

It remains to prove that $\nabla^{0}(\gamma)$ is cancelled by the face operators $d_{t}$. This is clear if $t=0$ and we assume $t>0$. Let $I$ be a partition. If we have $t-1, t \in I(k)$ for some $k$, then $t-1, t \notin I^{\prime}(k)$ and it follows that $d_{t} \nabla_{I}(\gamma)$ vanishes. Now assume that $t-1, t$ belongs to different components of the partition $I$ (say $t-1 \in I(p)$, $t \in I(q)$ ). Define $I^{0}$ as the partition $I$ with $t-1$ and $t$ transposed (explicitly, $\left.t \in I^{0}(p), t-1 \in I^{0}(q)\right)$. In this manner, we have

$$
\sigma_{I^{0 \prime}(k)} \delta_{t}=\sigma_{I^{\prime}(k)} \delta_{t}, \forall k=1, \ldots, r, \quad \operatorname{sgn}\left(I^{0}\right)=-\operatorname{sgn}(I)
$$

and hence

$$
\operatorname{sgn}(I) d_{t} \nabla_{I}(\gamma)+\operatorname{sgn}\left(I^{0}\right) d_{t} \nabla_{I^{0}}(\gamma)=0 .
$$

If $I^{0}=\sigma^{*} I$ for some $\sigma \in S_{r}$, then we have necessarily $I(p)=\{t-1\}, I(q)=\{t\}$ and $\sigma=(t-1 t)$. But if $\# I(k)=\left|v_{(k)}^{i}\right|$ for $k=1, \ldots, r$, then this possibility is excluded, because by hypothesis $\# I(k) \geq 2$. Hence, the map $I \mapsto I^{0}$ permutes the orbits of the action of the symmetric group $S_{r}$. This completes the proof of the identity $d_{t} \nabla^{0}(\gamma)=0$.

2.2.15. Lemma. The natural transformation $\nabla_{*}^{0}: \Gamma(M, V) \longrightarrow T(M)_{*}(V)$ is compatible with the composition of symmetric sequences. Explicitly, the diagram

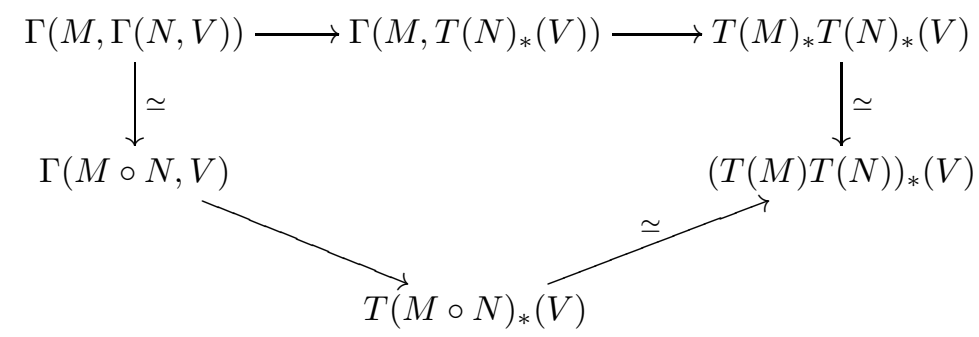

is commutative.

Proof. Fix $\gamma \in \Gamma(M, \Gamma(N, V))$.

At first, we calculate $\gamma^{\prime}$, the image of $\gamma$ in $T(M)_{*} T(N)_{*}(V)$, and $\gamma^{\prime \prime}$, the image of $\gamma$ in $(T(M) T(N))_{*}(V)$.

We may assume that $\gamma$ has an expansion of the form

$$
\gamma=\sum_{\sigma \in S_{n} / S_{\alpha}}(\sigma x) \otimes \sigma_{*}\left(\gamma_{1}^{\otimes \alpha_{1}} \cdots \gamma_{r}^{\otimes \alpha_{r}}\right)
$$


where $x \in M(n)^{S_{\alpha}}$, and $\gamma_{1}, \ldots, \gamma_{r} \in \Gamma(N, V)$. We use the same notation as in (1.1.20) for the expansion of $\gamma_{k}$ :

$$
\gamma_{k}=\sum y_{(k)} \otimes \underline{v}_{(k)} \in\left(N\left(s_{k}\right) \otimes V^{\otimes s_{k}}\right)^{S_{s_{k}}},
$$

where $y_{(k)} \in N\left(s_{k}\right)$ and $\underline{v}_{(k)} \in V^{\otimes s_{k}}$.

By definition of $\nabla^{0}$, using the fact 2.2.6, for $\gamma^{\prime} \in \pi_{*}\left(T\left(M, K \pi_{*} T(N, V)\right)\right)$, we obtain the expansion

$$
\gamma^{\prime}=\sum_{I \in \Pi(n) / S_{\alpha}} x \otimes \nabla_{I}\left(\nabla^{0}\left(\gamma_{1}\right)^{\otimes \alpha_{1}} \cdots \nabla^{0}\left(\gamma_{r}\right)^{\otimes \alpha_{r}}\right) .
$$

Let us calculate $\gamma^{\prime \prime} \in \pi_{*}(T(M \circ N, V))$. We denote $t=\alpha_{1} s_{1}+\cdots+\alpha_{r} s_{r}$. The wreath product $S_{\alpha_{1}} \prec S_{s_{1}} \times \cdots \times S_{\alpha_{r}} \prec S_{s_{r}}$ is the semi-direct product

$$
S_{\alpha_{1}} \cdot S_{s_{1}}^{\times \alpha_{1}} \times \cdots \times S_{\alpha_{r}} \cdot S_{s_{r}}^{\times \alpha_{r}}
$$

We have a canonical embedding

$$
S_{\alpha_{1}} \prec S_{s_{1}} \times \cdots \times S_{\alpha_{r}} \prec S_{s_{r}} \longleftrightarrow S_{t},
$$

which maps $\sigma \in S_{\alpha_{k}}$ to the obvious block-permutation. By concatenation, we obtain the word

$$
\underline{v}_{(1)}^{\otimes \alpha_{1}} \cdots \underline{v}_{(r)}^{\otimes \alpha_{r}} \in V^{\otimes t}
$$

The image of $\gamma$ under the canonical isomorphism

$$
\Gamma(M, \Gamma(N, V)) \longrightarrow \Gamma(M \circ N, V)
$$

has the expansion

$$
\sum\left[x \otimes \sigma \cdot\left(y_{(1)}^{\otimes \alpha_{1}} \cdots y_{(r)}^{\otimes \alpha_{r}}\right)\right] \otimes \sigma_{*}\left[\underline{v}_{(1)}^{\otimes \alpha_{1}} \cdots \underline{v}_{(r)}^{\otimes \alpha_{r}}\right]
$$

where $\sigma$ ranges over $S_{t} / S_{\alpha_{1}} 2 S_{s_{1}} \times \cdots \times S_{\alpha_{r}} 2 S_{s_{r}}$. Hence, by definition of $\nabla^{0}$, using the fact 2.2.6, we obtain

$$
\gamma^{\prime \prime}=\sum x \otimes\left(y_{(1)}^{\otimes \alpha_{1}} \cdots y_{(r)}^{\otimes \alpha_{r}}\right) \otimes \nabla_{I}\left(\underline{v}_{(1)}^{\otimes \alpha_{1}} \cdots \underline{v}_{(r)}^{\otimes \alpha_{r}}\right),
$$

where $I$ ranges over $\Pi(r) / S_{\alpha_{1}} 2 S_{s_{1}} \times \cdots \times S_{\alpha_{r}} \prec S_{s_{r}}$.

We now prove that $\gamma^{\prime}$ and $\gamma^{\prime \prime}$ correspond under the associativity isomorphism

$$
a_{T(M), T(N)}(V): T(M)_{*} T(N)_{*}(V) \stackrel{\simeq}{\longrightarrow}(T(M) T(N))_{*}(V) .
$$

We shall use the fact 2.1.6. Let $C$ be the graded module freely generated by $e_{1}, \ldots, e_{r}$, with $\left|e_{1}\right|:=\left|\gamma_{1}\right|, \ldots,\left|e_{r}\right|:=\left|\gamma_{r}\right|$. Let $c: C \longrightarrow N(T(N, K V))$ be the morphism, which maps $e_{i}$ to $\nabla^{0}\left(\gamma_{i}\right)$, for $i=1, \ldots, r$. As in $(2.1 .6), c^{\prime}: C \longrightarrow$ $\pi_{*}(T(N, K V))$ is the map induced in homotopy, and $c^{\prime \prime}: K C \longrightarrow T(N, K V)$ denotes the associated simplicial morphism. Then, $c^{\prime}$ induces

$$
c_{*}^{\prime}: \pi_{*}(T(M, K C)) \longrightarrow \pi_{*}\left(T\left(M, K \pi_{*} T(N, K V)\right)\right),
$$

and $c^{\prime \prime}$ induces

$$
c_{*}^{\prime \prime}: \pi_{*}(T(M, K C)) \longrightarrow \pi_{*}(T(M, T(N, K V))) .
$$

Consider the element $e \in \pi_{*}(T(M, K C))$ represented by the cycle

$$
\sum_{I \in \Pi(n) / S_{\alpha}} x \otimes \nabla_{I}\left(e_{1}^{\otimes \alpha_{1}} \cdots e_{r}^{\otimes \alpha_{r}}\right) .
$$


On one hand, we have

$$
c_{*}^{\prime}(e)=\sum_{I \in \Pi(n) / S_{\alpha}} x \otimes \nabla_{I}\left(\nabla^{0}\left(\gamma_{1}\right)^{\otimes \alpha_{1}} \cdots \nabla^{0}\left(\gamma_{r}\right)^{\otimes \alpha_{r}}\right) .
$$

Hence, $c_{*}^{\prime}(e)=\gamma^{\prime}$. On the other hand, we have:

$$
c_{*}^{\prime \prime}(e)=\sum x \otimes\left(y_{(1)}^{\otimes \alpha_{1}} \cdots y_{(r)}^{\otimes \alpha_{r}}\right) \otimes \nabla_{I}\left(\nabla_{J_{1}}\left(\underline{v}_{(1)}^{\otimes \alpha_{1}}\right) \otimes \cdots \otimes \nabla_{J_{r}}\left(\underline{v}_{(r)}^{\otimes \alpha_{r}}\right)\right),
$$

where $I$ ranges over $\Pi(n) / S_{\alpha}$ and $J_{k}, k=1, \ldots, r$, ranges over $\Pi\left(s_{k}\right) / S_{s_{k}}$. Then, using the fact 2.2.4, it is easy to see that $c_{*}^{\prime \prime}(e)=\gamma^{\prime \prime}$. Finally, we can deduce the identity

$$
a_{T(M), T(N)}(V)\left(\gamma^{\prime}\right)=\gamma^{\prime \prime}
$$

from the fact 2.1.6.

\section{REFERENCES}

1. M. Barr, C. Wells, Toposes, Triples, Theories, Grund. der Math. Wiss. 278, Springer Verlag, 1985. MR 86f: 18001

2. A.K. Bousfield, Homogeneous functors and their derived functors, Unpublished manuscript, Brandeis University (1967).

3. - Operations on derived functors of non-additive functors, Unpublished manuscript, Brandeis University (1967).

4. A.K. Bousfield, E.B. Curtis, D.M. Kan, D.G. Quillen, D.L. Rector, J.W. Schlesinger, The mod $-p$ lower central series and the Adams spectral sequence, Topology 5 (1966), 331-342. MR 33:8002

5. H. Cartan, Algèbres d'Eilenberg-MacLane et homotopie, Séminaire Henri Cartan, 1954-55.

6. F. Cohen, The homology of $\mathcal{C}_{n+1}$-spaces, $n \geq 0$, The homology of iterated loop spaces, Lecture Notes in Math. 533, Springer-Verlag, 1976, pp. 207-351. MR 55:9096

7. E.B. Curtis, Simplicial homotopy theory, Advances in Math. 6 (1971), 107-209. MR 42:3785

8. A. Dold, Homology of symmetric products and of other functors of complexes, Ann. of Math. 68 (1958), 54-80. MR 20:3537

9. MR 23:A646

10. A. Dold, D. Puppe, Homologie nicht-additiver funktoren, Ann. Inst. Fourier Grenoble 11 (1961), 201-312. MR 27:186

11. W. G. Dwyer, Homotopy operations for simplicial commutative algebras, Trans. Amer. Math. Soc. 260 (1980), 421-435. MR 81e:55027

12. - Higher divided squares in second-quadrant spectral sequences, Trans. Amer. Math. Soc. 260 (1980), 437-447. MR 81f:55022

13. B. Fresse, Opérations de Cartan pour les algèbres simpliciales sur une opérade, C. R. Acad. Sci. Paris Sér. I Math. 325 (1997), 247-252. MR 98c:18009

14. Cogroups in algebras over an operad are free algebras, Comment. Math. Helv. 73 (1998), 637-676. CMP 98:17

15. E. Getzler, J.D.S. Jones, Operads, homotopy algebra and iterated integrals for double loop spaces, preprint (1994).

16. V. Ginzburg, M.M. Kapranov, Koszul duality for operads, Duke Math. J. 76 (1994), 203-272. MR 96a:18004

17. P.G. Goerss, On the André-Quillen cohomology of commutative $\mathbf{F}_{2}$-algebras, Astérisque 186, 1990. MR 92b:18012

18. P.G. Goerss, T.J. Lada, Relations among homotopy operations for simplicial commutative algebras, Proc. Amer. Math. Soc. 123 (1995), 2637-2641. MR 95k:18007]

19. H.-W. Henn, J. Lannes, L. Schwartz, The categories of unstable modules and unstable algebras over the Steenrod algebra modulo nilpotent objects, Amer. J. Math. 115 (1993), 1053-1106. MR 94i:55024

20. M. Hovey, B. Shipley, J. Smith, Symmetric spectra, preprint (1998).

21. N. Jacobson, Lie algebras, Interscience Tracts in Pure and Applied Math. 10, Interscience Publishers, 1962. MR 26:1345 
22. S. Mac Lane, Homology, Grund. der Math. Wiss. 114, Springer Verlag, 1963.

23. - Categories for the Working Mathematician, Second Edition, Graduate Texts in Math. 5, Springer Verlag, 1998. MR 50:7275

24. M. Markl, Distributive laws and Koszulness, Ann. Inst. Fourier 46 (1996), 307-323. MR 97i: 18008

25. J.P. May, Simplicial objects in algebraic topology, Van Nostrand, New York, 1967. MR 36:5942

26. - The geometry of iterated loop spaces, Lecture Notes in Math. 271, Springer-Verlag, 1972. MR 54:8623b

27. C. Reutenauer, Free Lie Algebras, London Math. Soc. Monographs 7, Clarendon Press, 1993. MR 94j:17002

28. N. Roby, Construction de certaines algèbres à puissances divisées, Bull. Soc. Math. France 96 (1968), 97-113. MR 38:2164

29. J.M. Turner, Relations in the homotopy of simplicial abelian Hopf algebras, J. Pure Appl. Algebra 134 (1999), 163-206. CMP 99:06

30. Operations and spectral sequences. I, Trans. Amer. Math. Soc. 350 (1998), 3815-3835. MR 98m:18005

Laboratoire J.A. Dieudonné, Université de Nice, Parc Valrose, F-06108 Nice Cedex 02, FRANCE

E-mail address: fresse@math.unice.fr 